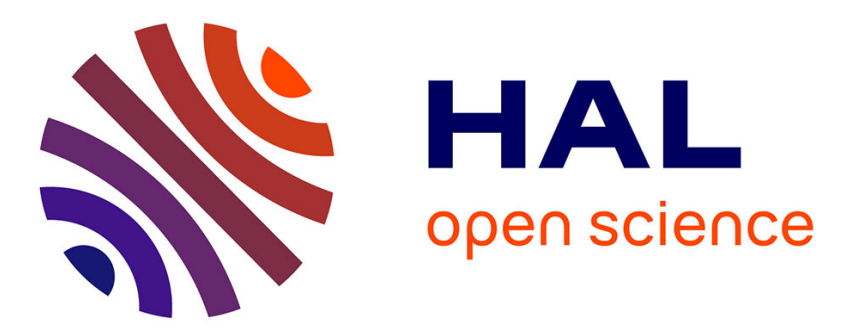

\title{
Reconstruction of tomographic images from limited range projections using discrete Radon transform and Tchebichef moments
}

\author{
Xiubin Dai, Huazhong Shu, Limin M. Luo, Guo-Niu Han, Jean-Louis \\ Coatrieux
}

\section{To cite this version:}

Xiubin Dai, Huazhong Shu, Limin M. Luo, Guo-Niu Han, Jean-Louis Coatrieux. Reconstruction of tomographic images from limited range projections using discrete Radon transform and Tchebichef moments. Pattern Recognition, 2010, 43 (3), pp.1152-1164. 10.1016/j.patcog.2009.07.009 . inserm00421043

\section{HAL Id: inserm-00421043 https://www.hal.inserm.fr/inserm-00421043}

Submitted on 30 Sep 2009

HAL is a multi-disciplinary open access archive for the deposit and dissemination of scientific research documents, whether they are published or not. The documents may come from teaching and research institutions in France or abroad, or from public or private research centers.
L'archive ouverte pluridisciplinaire HAL, est destinée au dépôt et à la diffusion de documents scientifiques de niveau recherche, publiés ou non, émanant des établissements d'enseignement et de recherche français ou étrangers, des laboratoires publics ou privés. 
Reconstruction of tomographic images from limited range projections using discrete Radon transform and Tchebichef moments

X.B. Dai ${ }^{\text {a, e }}$, H.Z. Shu ${ }^{\text {a, e, }}{ }^{*}$, L.M. Luo ${ }^{\text {a, e }}$, G.N. Han ${ }^{\text {b }, ~ J . L . ~ C o a t r i e u x ~}{ }^{\text {c, d, e }}$

${ }^{a}$ Laboratory of Image Science and Technology, School of Computer Science and Engineering,

Southeast University, 210096, Nanjing, China

${ }^{\mathrm{b}}$ IRMA, Université Louis Pasteur et C.N.R.S., 7, rue René-Descartes F, 67084 Strasbourg,

France

${ }^{\mathrm{c} I N S E R M, ~ U 642, ~ R e n n e s, ~ F-35000, ~ F r a n c e ~}$

${ }^{\mathrm{d}}$ Université de Rennes 1, LTSI, Rennes, F-35000, France

${ }^{\mathrm{e}}$ Centre de Recherche en Information Biomédicale Sino-Français (CRIBs)

Information about the corresponding author

Huazhong Shu, Ph. D

Laboratory of Image Science and Technology

Department of Computer Science and Engineering

Southeast University, 210096, Nanjing, China

Tel: 00-86-25-83 794249

Fax: 00-86-25-83 792698

Email: shu.list@seu.edu.cn 
Abstract: This paper presents an image reconstruction method for X-ray tomography from limited range projections. It makes use of the discrete Radon transform and a set of discrete orthogonal Tchebichef polynomials to define the projection moments and the image moments. By establishing the relationship between these two sets of moments, we show how to estimate the unknown projections from known projections in order to improve the image reconstruction. Simulation results are provided in order to validate the method and to compare its performance with some existing algorithms.

Keywords: discrete Radon transform; discrete orthogonal moments; projection moments; image reconstruction

\section{Introduction}

The issue of image reconstruction has received much attention in the medical imaging literature. This is due to the constant search for improvements of imaging modalities, ranging from X-ray computerized tomography and emission tomography up to acoustic and optical techniques. They all bring different insights in the human body either morphological or functional. The standard mathematical model of X-ray computerized tomography (CT) assumes that the sensing device measures the line integrals of the object attenuation coefficient at some known orientations. An analytical formulation for the reconstruction of two-dimensional (2-D) tomographic images from projections, i.e., an inverse problem, has been first proposed by Radon in 1917. The filtered back-projection (FBP) algorithm, which can be seen as a computer implementation of Radon's inversion formula, still plays an important role although algebraic methods are also intensively used [1-3]. However, the reconstruction based on FBP algorithm requires the projections for all angles from 0 to $\pi$. A major health concern today is related to the reduction of dose to the patient which means limiting either the X-ray source intensity or the number of projections. This issue is critical not only for diagnosis imaging but also in interventional setting where for instance rotational $-\mathrm{X}$ is used.

One way to improve the quality of the reconstructed image when only limited range projections are available consists to estimate the projections at unknown views by moment-based approaches [4, 
5]. Milanfar et al. [6] presented a variational framework for the tomographic reconstruction of an image from the maximum likelihood estimates of its orthogonal moments. Basu and Bresler [7, 8] investigated the problem of recovering the view angles from the projection data by means of moment method. By establishing a relationship between the image geometric moments and projection moments, Wang and Sze [9] presented an approach to reconstruct the CT images from limited range projections. Shu et al. [10] extended Wang's method by using the orthogonal Legendre moments to improve the quality of the reconstructed image. Its advantage is that the orthogonal moments have simple inverse transform, thus the image can be more easily reconstructed from the orthogonal moments. Moreover, the geometric moments, especially at high order, are sensitive to noise and digitization error. However, both Wang's and Shu's methods were based on the use of continuous moments. When applied to 2-D digital images, the double integrals are usually approximated by discrete summations that lead to numerical errors in the computed moments. The discrete orthogonal moments recently introduced for image analysis [11-14], in particular Tchebichef moments, have shown a better image representation capability than the traditional continuous orthogonal moments because they do not require any discrete approximation for numerical implementation. Two other arguments motivate our use of the discrete orthogonal Tchebichef polynomials: (1) they are the simplest among all the discrete orthogonal polynomials; (2) they have a definition domain ideally suited for square images [11].

Since the reconstruction techniques are typically modeled in the domain of the continuous 2-D plane from which projections are acquired, the need to impose the reconstructed solution as an image on a 2-D discrete grid of pixels is usually accommodated at a later stage of the implementation for each algorithm. In some approaches, these discrete sampling issues do constrain the data acquisition process and the reconstruction and may influence the experimental design of CT scanner. Moreover, when using the discrete orthogonal polynomials to define the projection moments, the traditional Radon transform is no longer applicable, and a discrete version of Radon transform is required. Guédon and Normand [15] introduced the so-called Mojette transform as a discrete geometric tool. Beylkin [16] described the discrete Radon transform (DRT) to map a set of sampled image points 
onto a set of discrete projections. The algebraic mapping for this transformation can be computed exactly since no interpolation of the data is required. Matus and Flusser [17] developed a group theoretic and Fourier based approach to the DRT. Svalbe [18] and Kingston [19] derived improved versions of DRT to handle both continuous projections and discrete projections in Fourier and Radon space. The advantage of the techniques reported in [18] and [19] is that they require no pre-processing of the projection data, a straightforward 1-D linear interpolation and a simple sorting of projection samples.

The organization of this paper is as follows. A brief outline of the discrete Radon transform is provided in Section 2. The definition of projection moments and image moments is given in Section 3. In this section, we also establish a relationship between the projection moments and image moments and discuss how to estimate the projection moments at any specific view from the image moments. The performance of the proposed solution with a comparison to some existing methods is reported in Section 4. The concluding remarks are given in Section 5.

\section{Discrete Radon transform}

Because the data projections are acquired in discrete form, we use the following version of DRT suggested by Kingston [19]

$$
\begin{gathered}
R(k, \theta)=\sum_{x=0}^{N-1} \sum_{y=0}^{N-1} I(x, y) \delta\left(k-x y_{\theta}+y x_{\theta}\right) \\
\theta=\tan ^{-1}\left(x_{\theta} / y_{\theta}\right), \quad x_{\theta} \in Z, y_{\theta} \in Z
\end{gathered}
$$

where $I(x, y)$ is the image function, $N \times N$ is the image size, and $N$ is assumed to be a prime number; $\delta(x)$ is the delta function, $k \in\left\{0,1,2 \ldots N_{\theta}-1\right\}, N_{\theta}=N\left(\left|x_{\theta}\right|+y_{\theta}\right), x_{\theta}$ and $y_{\theta}$ are respectively the horizontal and vertical distances with the nearest pixels.

Using the Fourier slice theorem, the mapping from a continuous projection to a discrete projection can be implemented by interpolation. It means that each individual discrete projection $R(k, \theta)$ at a given view $\theta$, denoted hereafter by $R_{\theta}(k)$, can be calculated by interpolation using the FFT data of all continuous projections $g_{\theta}(s)$, which can be referred to Kingston [19]. The image can be reconstructed using the inverse DRT (IDRT) 


$$
I(x, y)=\frac{1}{N}\left[\sum_{m=0}^{N} R\left(\langle x-m y\rangle_{\bmod (N)}, m\right)-\sum_{\lambda=0}^{N-1} R(\lambda, 0)\right]
$$

where $R(\lambda, m)$ is another version of DRT which is given by [18]

$$
R(\lambda, m)=\left\{\begin{array}{l}
\sum_{x=0}^{N-1} I(x, \lambda), \quad \text { for } m=0, \\
\sum_{y=0}^{N-1} I\left(\langle m y+\lambda\rangle_{\bmod (N)}, y\right), \quad \text { for } 0<m \leq N .
\end{array}\right.
$$

Here $<A>\bmod (N)$ denotes the residue of $A$ modulo $N, t \in\{0,1,2, \ldots, N-1\}$.

If only the projections $R(k, \theta)$ defined by Eq. (1) are available, we need to transform them into the corresponding $R(\lambda, m)$ before reconstruction. The detailed description of transformation from $R(\lambda, m)$ to $R(k, \theta)$ can be found in [18] and [19].

\section{Method}

\subsection{Discrete projection moment and image moment}

The moments of discrete projection $R_{\theta}(k)$ are called the discrete projection moments. In this paper, we use a set of discrete orthonormal polynomials to define the projection moments. Let $t_{p}(k), p=0,1$, $\ldots, L$, be a set of discrete orthonormal polynomials defined on the interval $\left[0, N_{\theta}-1\right]$, the $p$ th order orthonormal projection moments of $R_{\theta}(k)$ is defined as

$$
H_{p}(\theta)=\sum_{k=0}^{N_{\theta}-1} t_{p}(k) R_{\theta}(k) .
$$

Substituting (1) into (4) and using the property of delta function, we have

$$
H_{p}(\theta)=\sum_{x=0}^{N-1} \sum_{y=0}^{N-1} I(x, y) t_{p}\left(y_{\theta} x-x_{\theta} y\right) .
$$

Let $T_{n m}$ be the $(n+m)$ th order discrete orthonormal moment of the image intensity function $I(x, y)$ defined as

$$
T_{n m}=\sum_{x=0}^{N-1} \sum_{y=0}^{N-1} t_{n}(x) t_{m}(y) I(x, y), \quad 0 \leq n, m \leq N-1
$$

The orthogonality property of polynomials leads to the following approximate inverse moment 
transform

$$
\hat{I}(x, y)=\sum_{n=0}^{M} \sum_{m=0}^{n} T_{n-m, m} t_{n-m}(x) t_{m}(y),
$$

where $M$ denotes the maximum order of moments used in the reconstruction.

The objective of the next subsection is to establish a relationship between the orthonormal projection moments defined by (5) and the discrete orthonormal moments of $I(x, y)$ defined by $(6)$.

\subsection{Relationship between Discrete projection moment and image moment}

Let us first introduce some basic definitions. Define the $p$ th order discrete orthonormal polynomial $t_{p}(x)$ as

$$
t_{p}(x)=\sum_{q=0}^{p} c_{p q}(-x)_{q},
$$

where $(-x)_{q}$ is the Pochhammer symbol defined by

$$
(-x)_{q}=(-x)(-x+1)(-x+2) \cdots(-x+q-1), \quad \text { for } q \geq 1
$$

with $(-x)_{0}=1$.

It can be deduced from (8) that

$$
(-x)_{l}=\sum_{r=0}^{l} d_{l r} t_{r}(x) \quad \text { for } 0 \leq l \leq p,
$$

where $D_{p}=\left(d_{l q}\right)$, with $0 \leq q \leq l \leq p$, is the inverse matrix of the lower triangular matrix $C_{p}=\left(c_{l q}\right)$. Now we can establish the relationship between the projection moments and image moments.

Theorem 1. The discrete orthogonal projection moment of order $p$ at given view $\theta, H_{p}(\theta)$, can be expressed as a linear combination of discrete orthogonal image moments, $T_{n m}$, of same order and lower, i.e.,

$$
H_{p}(\theta)=\sum_{n=0}^{p} \sum_{m=0}^{p-n} \mu_{n m}(p, \theta) T_{n m},
$$

where 


$$
\begin{aligned}
\mu_{n m}(p, \theta)= & \sum_{r=0}^{p-n-m} \sum_{s=0}^{n+r} \sum_{q=n+m+r}^{p} \sum_{i=n}^{n+r-s} \sum_{j=m}^{n+m+r-i}(-1)^{q+s+i+j-r-n} c_{p q} d_{i n} d_{j m}\left(\begin{array}{c}
n+m+r \\
s+m
\end{array}\right) \\
\cdot & S_{1}(q, n+m+r) S_{2}(n+r-s, i) S_{2}(m+s, j) y_{\theta}^{m+s} x_{\theta}^{n+r-s}
\end{aligned}
$$

Here $S_{1}(i, j)$ and $S_{2}(i, j)$ are respectively the first kind and second kind of Stirling numbers [20].

The proof of Theorem 1 is given in Appendix.

Equation (10) can be expressed in matrix form. Let $H_{M}(\theta)=\left[H_{0}(\theta), H_{1}(\theta), \ldots, H_{M}(\theta)\right]^{T}, T^{(k)}=\left[T_{k 0}\right.$, $\left.T_{k-1,1}, T_{k-2,2}, \ldots, T_{1, k-1}, T_{0 k}\right], \Phi_{M}=\left[T^{(0)}, T^{(1)}, T^{(2)}, \ldots, T^{(M)}\right]^{T}$ where $M$ is the maximum order of moments to be used, then we have

$$
H_{M}(\theta)=\Psi_{M}(\theta) \Phi_{M}
$$

where $\Psi_{M}(\theta)$ denotes a matrix of size $(M+1) \times(M+1)(M+2) / 2$ which is defined by

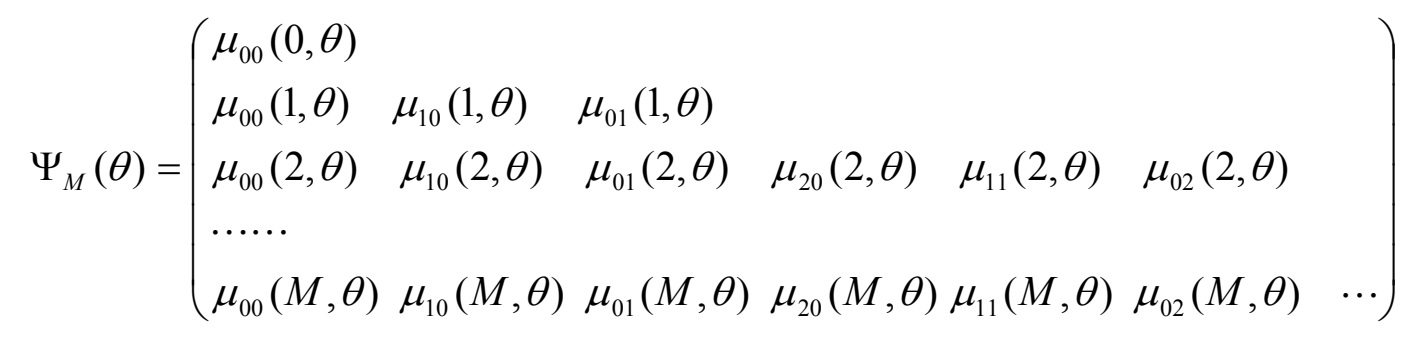

Here the elements $\mu_{n m}(p, \theta)$, with $0 \leq n, m, p \leq M$, are given by (11).

Proposition 1. Given the discrete projections of $I(x, y)$ at $L$ different sampling angles $\theta_{i}, 1 \leq i \leq L$, one can uniquely determine the first $L$ moment vectors $T^{(k)}$ of $I(x, y), 0 \leq k<L$. This can be done using only the first $L$ orthogonal discrete projection moments.

For the proof of Proposition 1, we refer to Milanfar et al. [6].

Theorem 1 describes a general result which is valid for all the sets of discrete orthonormal polynomials. In the rest of the paper, we focus on the use of discrete orthonormal Tchebichef polynomials. Note that other discrete orthogonal polynomials such as Krawtchouk [12], Racah [13] or dual Hahn polynomials [14] can be considered. The $p$ th order discrete orthonormal Tchebichef polynomial is defined by [11]

$$
t_{p}(x)=\frac{(1-N)_{p}}{\beta(p, N)}{ }_{3} F_{2}(-p,-x, 1+p ; 1,1-N ; 1)=\sum_{r=0}^{p} c_{p r}(-x)_{r}, \quad x=0,1, \ldots, N-1,
$$

where ${ }_{3} F_{2}(\cdot)$ is the generalized hypergeometric function, $\beta(p, N)$ is a constant independent of $x$. A 
suitable choice of $\beta(p, N)$ is [11]

$$
\beta(p, N)=\sqrt{(2 p) !\left(\begin{array}{c}
N+p \\
2 p+1
\end{array}\right)}
$$

and $c_{p r}$ is given by

$$
c_{p r}=\frac{(-1)^{r}}{\beta(p, N)} \frac{(p+r) !}{(p-r) !(r !)^{2}} \frac{(1-N)_{p}}{(1-N)_{r}} .
$$

An essential step when applying a given polynomial to the reconstruction problem is to find the inverse matrix $D_{p}$. For the discrete orthonormal Tchebichef polynomials, we have the following result.

Proposition 2. For the lower triangular matrix $C_{p}$ whose elements $c_{l q}$ are defined by (16), the elements of the inverse matrix $D_{p}$ are given by

$$
d_{l q}=(-1)^{q} \frac{\beta(q, N)(2 q+1)(l !)^{2}(1-N)_{l}}{(l+q+1) !(l-q) !(1-N)_{q}}, \quad 0 \leq q \leq l \leq p .
$$

The proof of Proposition 2 is given in Appendix.

From the above Proposition, we can derive an explicit expression of $\mu_{n m}(p, \theta)$ in (11) for discrete normalized Tchebichef polynomials. Let

$$
v_{r s q i j}(p, n, m)=c_{p q} d_{i n} d_{j m}\left(\begin{array}{c}
n+m+r \\
s+m
\end{array}\right) S_{1}(q, n+m+r) S_{2}(n+r-s, i) S_{2}(m+s, j),
$$

then (11) becomes

$$
\mu_{n m}(p, \theta)=\sum_{r=0}^{p-n-m} \sum_{s=0}^{n+r} \sum_{q=n+m+r}^{p} \sum_{i=n}^{n+r-s} \sum_{j=m}^{n+m+r-i}(-1)^{i+j+q+s-r-n} v_{r s q i j}(p, n, m) y_{\theta}^{m+s} x_{\theta}^{n+r-s} .
$$

Substitution of (16) and (17) into (19) leads to

$$
\mu_{n m}(p, \theta)=R \sum_{r=0}^{p-n-m} \sum_{s=0}^{n+r} Z_{1} Z_{2} Z_{3} y_{\theta}^{m+s} x_{\theta}^{n+r-s}
$$

where

$$
R=\sqrt{\frac{(2 p+1)(2 n+1)(2 m+1)(N+n) !(N+m) !(N-n-1) !(N-m-1) !}{(N+p) !(N-p-1) !}},
$$




$$
\begin{gathered}
Z_{1}=\frac{(n+m+r) !}{(m+s) !(r+n-s) !}, \\
Z_{2}=\sum_{q=n+m+r}^{p}(-1)^{p+q+s-r-n} U_{q}(p, N) S_{1}(q, n+m+r), \\
U_{q}(p, N)=\frac{(p+q) !(N-q-1) !}{(p-q) !(q !)^{2}}, \\
Z_{3}=\sum_{i=n}^{r-s+n} \sum_{j=m}^{r+n+m-i} W_{i j}(n, m, N) S_{2}(r-s+n, i) S_{2}(s+m, j), \\
W_{i j}(n, m, N)=\frac{(i !)^{2}(j !)^{2}}{(i+n+1) !(i-n) !(m+j+1) !(j-m) !(N-i-1) !(N-j-1) !} .
\end{gathered}
$$

The coefficients $U_{q}(p, N)$ and $W_{i j}(n, m, N)$ can be computed through

$$
\begin{aligned}
& U_{q+1}(p, N)=\frac{(p+q+1)(p-q)}{(N-q-1)(q+1)^{2}} U_{q}(p, N), \quad \text { for } q \geq n+m+r, \\
& W_{i+1, j}(n, m, N)=-\frac{(i+1)^{2}(N-i-1)}{(i+n+2)(i-n+1)} W_{i, j}(n, m, N), \quad \text { for } i \geq n, \\
& W_{i, j+1}(n, m, N)=-\frac{(j+1)^{2}(N-j-1)}{(m+j+2)(j-m+1)} W_{i j}(n, m, N), \quad \text { for } j \geq m,
\end{aligned}
$$

with

$$
\begin{aligned}
& U_{n+m+r}(p, N)=\frac{(p+n+m+r) !(N-n-m-r-1) !}{(p-n-m-r) !((n+m+r) !)^{2}}, \\
& W_{n m}(n, m, N)=\frac{(n !)^{2}(m !)^{2}}{(2 n+1) !(2 m+1) !(N-n-1) !(N-m-1) !} .
\end{aligned}
$$

Note that the first and second kinds of Stirling numbers, $S_{1}(r, k)$ and $S_{2}(k, m)$, can be pre-calculated and stored in a look-up table. The above recurrence formulas allow reducing the computational complexity of $\mu_{n m}(p, \theta)$.

\subsection{Estimation of the discrete projection moments at any view}

Based on the relationship between the given projection moments and the image moments established above, the following shows how to compute the unknown discrete projections from the 
image moments.

The orthogonality of Tchebichef polynomials leads to the following approximate inverse transform of (4)

$$
\widehat{R}_{\theta}(k)=\sum_{p=0}^{M} H_{p}(\theta) t_{p}(k)
$$

where $M$ denotes the maximum order of moments used in the reconstruction.

Substituting Eq. (10) into Eq. (32), we have

$$
\widehat{R}_{\theta}(k)=\sum_{p=0}^{M} t_{p}(k) \sum_{n=0}^{p} \sum_{m=0}^{p-n} \mu_{n m}(p, \theta) T_{n m} .
$$

Equation (33) provides the basis to estimate the unknown discrete projections from the known image moments. When all the projection data are available, they can be used to reconstruct the original image. The main steps of the reconstruction process are summarized as follows.

1) Acquire the discrete projections from corresponding known continuous projections according to the method described in Section 2;

2) Compute the discrete projection moments up to order $M$ from given discrete projections using $(4)$;

3) Calculate the image moments of order up to $M$ based on (12);

4) Estimate the unknown discrete projections from image moments using (33);

5) Once the unknown projections are estimated, use either the FBP method or the inverse discrete Radon transform (IDRT) defined by (2) to reconstruct the original discretized image $I(x, y)$.

It is worth noting that the image reconstruction can also be done by using the approximate inverse moment transform defined by (7) when the image moments of order up to $M$ are calculated. If such a strategy is adopted, the steps 4) and 5) can be omitted. However, we prefer to use the technique described above. The reason for such a choice will be explained in the next section.

\section{Results and discussions}

\subsection{Simulation}

A simulated image of size $127 \times 127$ pixels (Fig. 1) has been built for performance evaluation and 
comparison purpose. The main object is an ellipse with size $42 \times 39$ pixels and the two internal ellipses have $6 \times 5$ and $9.5 \times 9$ axes (The three ellipses will be denoted by E1, E2 and E3 whose density values are 1,3 and 4 respectively). We use this phantom to compare the two methods for computing the image moments. The first method is directly based on (7) since the image is known and the second one is performed through the computation of discrete projection moments with the help of (12). In both methods, the discrete orthonormal moments, $T_{n m}$, of order up to 16 are calculated. The differences between the moment values using these two methods are shown in Table 1. It can be observed from this table that the difference between the real image moments and estimated image moments is very small. This result points out that the image moments can be well estimated from the discrete projection moments.

To test the robustness of the proposed method, we first consider the case where the projections are known over the whole interval $[0, \pi]$, the reference axis being horizontal. The image moments of order up to $M=20$ are calculated, and (33) is used to estimate the projection, $\widehat{R}_{\theta}(k)$, at any specified view $\theta$. The initially acquired projections $\mathrm{g}_{\theta}(\mathrm{s})$ and the projections estimated with the methods based on both the Legendre moments (denoted by $\left.\widetilde{g}_{\theta}(s)\right)$ and the Tchebichef moments of order up to $M=20$ at angles $0^{\circ}, 40^{\circ}, 60^{\circ}, 90^{\circ}, 130^{\circ}$ and $140^{\circ}$ are depicted in Fig. 2. Note that for comparison purpose, the estimated discrete projections $\widehat{R}_{\theta}(k)$ have been converted to $\bar{g}_{\theta}(s)$ using the interpolation in Fourier space [19]. They show a good fit between the estimated and the original projections, with a slightly better performance of the proposed method when compared to the Legendre moment based method, as exemplified for the angles $40^{\circ}$ and $140^{\circ}$. We then assume that projections are available in the range of $25^{\circ} \leq \theta \leq 155^{\circ}$, and unknown over $0^{\circ} \leq \theta<25^{\circ}$ and $155^{\circ}<\theta \leq 180^{\circ}$. Fig. 3 shows the estimated projections using both the proposed method and Legendre moment-based method at views of $0^{\circ}, 10^{\circ}, 30^{\circ}, 150^{\circ}, 170^{\circ}$ and $180^{\circ}$. Here also, the discrete Tchebichef moments perform better than the continuous Legendre moments.

We now consider the problem of image reconstruction from limited range projections. The following configuration of continuous projections is used: (a) the total view varies from $25^{\circ}$ to $155^{\circ}$; (b) the angular sampling rate is $1^{0}$; (c) the spatial ray sampling rate is 127 rays per view. Fig. 4(b) 
shows the reconstructed image from incomplete projections $\left(25^{0}-155^{0}\right)$ using the filtered back-projection (FBP) algorithm. Because the FBP algorithm requires the continuous projections, $g(s$, $\theta$ ), for all $s$ and $\theta$, the value of $g(s, \theta)$ is set to zero at the unknown views. The projection data considered here are assumed to be noiseless and a Ram-Lak filter with cutoff frequency $\xi_{\max } \geq 1 /(2 \Delta s)$ has been applied for the FBP in order to avoid the overlapping phenomenon ( $\Delta s$ denotes the sampling interval of $s$ ) [2]. Note also that for noiseless data, the value of $\xi_{\max }$ is chosen in such a way that the mean square error (MSE) between the original image $I$ and the reconstructed image $\hat{I}$ is minimal. The MSE is defined by

$$
\operatorname{MSE}(\%)=\frac{\|\hat{I}-I\|^{2}}{\|I\|^{2}} \times 100 \% \text {. }
$$

We now apply our method as well as the continuous orthogonal moment based method described in [10] to compute the image moments from the projection moments with maximum order $M$ equal to 5, 10, 15 and 20. These moment values are used to estimate the unknown projections. Then, the FBP method is applied to reconstruct the image from all the projections in which the missing angle projections are estimated by the method based on Legendre moments (Fig. 4(e)) and IDRT method is used to reconstruct the image where the unknown projections are estimated by Tchebichef moments (Fig. 4(f)). We also apply the FBP algorithm instead of the IDRT to reconstruct the image: the corresponding reconstructed images are depicted in Fig. 4(g). For comparison purpose, the reconstructed results using MLEM (Maximum Likelihood Expectation Maximum) [21], MXE (Minimum Cross-Entropy) [22] and SART (Simultaneous Algebraic Reconstruction Technique) [23] are displayed in Fig. 4 (b)-(d) where the results have been obtained after 100 iterations (20 iterations for SART). As previously indicated, when the image moments are available, it is also possible to use the inverse moment transform defined by (7) to reconstruct the original image. Fig. 4(h) shows the reconstructed images using (7) for $M=5,10,15$, and 20 respectively.

Table 2 shows the MSEs for the images displayed in Fig. 4 and Table 3 provides the mean grey levels of three reconstructed ellipses E1, E2 and E3. From Fig. 4, Tables 2 and 3, we can see that the statistical methods achieve better performance compared to FBP method except for ART method 
when parts of projection data are missing. That is because the comparison and correction steps in iterative statistical methods can modify the incomplete projections during each iteration. While they significantly decrease with the moment order, all the reconstructed results remain very close when using the different methods, even if the Tchebichef moments lead to lower MSEs. However, when looking at the images, different behaviors can be observed. If we find the classical artifacts of the FBP and statistical algorithms in (a)-(d), (e) and also in (g), they are not observed in (f) where the IDRT is applied. The contours of the phantom ellipses are better defined and their original shapes are preserved. Conversely, we can see a loss in homogeneity of the regions, with a random like pattern, without any obvious link with the acquisition geometry. The Legendre and Tchebichef based images do not visually show significant differences when they are associated with the FBP. It can also be seen from this figure that, for the same value of $M$, the quality of the reconstructed image based on (7) (shown in (h)) is poorer than those obtained with either FBP or IDRT algorithm. One way to improve the quality of the reconstructed image based on the inverse moment transform is to increase the value of $M$. However, such a strategy would lead to higher computational complexity since the dimension of the matrix $\Psi_{M}(\theta)$ defined in (13) is $(M+1) \times(M+1)(M+2) / 2$. Moreover, high order moments are more sensitive to noise than the low order moments [24] (this point will be discussed later). For these reasons, the inverse moment transform in the reconstruction process was not further considered. In this experiment, the whole procedure costs $35.9 \mathrm{~s}$ (35.11s when using FBP method for reconstruction), and the computation time required for each step (from step 2 to step 5 ) is respectively $2.6 \%, 51.8 \%$, $24.5 \%, 12.1 \%$ of the total time. Note that the program was implemented in Matlab 7.0.1 on a PC CORE $23.0 \mathrm{GHz}, 2.0 \mathrm{~GB}$ RAM. It is worth noting that the computation of the matrix $\Psi_{M}(\theta)$ for both known and unknown views in this experiment is approximately $73 \%$ of the whole computation time, thus, it is the most time consuming process in our method. Generally speaking, the greater value of $M$, the higher computation time will be.

To bring additional cues, we compare our method with the Legendre moment based method, using the maximum order of moments required to get similar values of MSE. In all cases (Table 4), the maximum order of moments used in the proposed method is lower than that needed for Legendre 
moments. Thus, a significant reduction in computational complexity can be reached.

To test the robustness of the methods to noise, the same example (the projections are always assumed to be available in the range of $25^{\circ} \leq \theta \leq 155^{\circ}$ ) is used where Poisson noise, with the mean value equal to the projection intensity, was added to the projection data. When the input data are corrupted by noise, the choice of the cut-off frequency may be more complicated. According to [25], a filter with a cut-off frequency that is too high may maintain resolution and contrast, but allow noise to degrade the reconstructed image quality. Conversely, a filter with a too low cut-off frequency will suppress image noise, but may oversmooth the image, decrease contrast and eventually introduce ringing artifacts. Different values of $\xi_{\max }$ have been tested in this experiment, the MSEs corresponding to the FBP method, the Legendre moment method associated with FBP algorithm, and the proposed method combined with both IDRT and FBP, are illustrated in Table 5. It can be observed from this table that for a fixed value of $M$, the MSEs depend on the cut-off frequency except for the case where the IDRT is used in the reconstruction process (i.e. no filtering is required for IDRT). Fig. 5 depicts the reconstructed images corresponding to different approaches for $\xi_{\max }=0.7$ and $M=5,10$, 15 and 17, respectively (in (e)-(g)), as well as the results obtained with the statistical methods after 50 iterations (in (b)-(d)). The corresponding MSE values are shown in Table 6. We can see from Figure 5 and Table 6 that for $M=17$, the quality of the reconstructed images degrade for both Legendre moment method and the proposed method. This effect is due to the fact that the higher order moments are sensitive to noise. These results point out that for higher noise level, lower moment order should be used. To improve the quality of the reconstruction in case of noise, one way consists to utilize the projection based filter technique to reduce its impact as suggested by Schaeffter et al. in [26].

\subsection{Evaluation on "pseudo-real" data}

Since real projection data are not available from imaging devices, the evaluation has been conducted on previously reconstructed images, acquired on standard CT devices (Fig. 6(a)) whose sizes are all 509*509, hence the so-called "pseudo-real" data. A parallel geometry (723 rays per view) has been used to generate the projections over $\left[0^{0}, 180^{\circ}\right]$ with $1^{0}$ angular sampling. Poisson noise with 
mean value equal to the projection intensity was added to the projections.

The limited range projections are defined over $\left[\alpha, 180^{\circ}-\alpha\right]$ where $\alpha$ is an adjustable parameter. The approach using the discrete Tchebichef moments has been compared to the continuous Legendre moment method for $\alpha=0,10,15,20,25$. These moment values are then used to estimate the unknown projections. The reconstructed images using different approaches for different values of $\alpha$ are displayed in Fig. 6. Fig. 6(b)-(f) and Fig. 6(g)-(k) provide the reconstruction results for different values of $\alpha$ using FBP method and the method based on Legendre moments. The cut-off frequency of the Ram-Lak filter used in FBP algorithm is set to 0.7. As in the previous example, when the unknown projections are estimated with Eq. (32), the IDRT method was applied. The reconstruction results are shown in Fig. 6 (1)-(p). These pictures confirm the observations previously made on the phantom data. The quality of the reconstructions using FBP degrades quickly with smaller intervals of view, especially on the left and right sides of the head. The resulting images remain blurred and a loss of contrast can be seen even for the lowest value of $\alpha$. However, some soft tissues features (for instance, the black regions at the center of the brain) are restituted. The reconstruction resulting from our approach provides a good contrast for the skull and an improved resolution for very fine bones (for instance just below the nose) with well defined contours. However, the soft tissue details mentioned before are lost and the regions depict a random-like texture. The corresponding MSE values are displayed in Table 7 and the difference of mean grey levels of region of interest (denoted by a rectangle in Fig. 6(a)) between the reconstructed results and the original image (Fig. 6(a)) are displayed in Table 8 . They show the fast decrease in performance when $\alpha$ is increasing for FBP. If this trend is similar for moment-based approaches, it is less pronounced. In other words, the moment-based approaches seem more robust to limited range projections. Here also, both the MSE and the difference of mean grey levels obtained with Tchebichef moments and IDRT are the lowest.

\section{Conclusion and Perspectives}

A new method has been described for tomographic image reconstruction from limited range projections. The discrete Radon transform and the discrete Tchebichef polynomials have been used to 
establish the relationship between the projection moments and image moments. Based on this relationship, the image moments were calculated from the known projections, and they were then used to estimate the unknown projections. The effectiveness of the method has been confirmed by the results obtained on simulated phantom and "pseudo-real" data. These experiments have shown some improvement in MSEs when using Tchebichef polynomials instead of the continuous orthogonal moments (e.g. Legendre moments). The visual inspection of the reconstructed images, however, pointed out advantages and disadvantages. On the positive side, the contours and small bone structures are better preserved. On the negative side, region features are less homogeneous and soft tissue properties can be lost. The CT scanner being mainly devoted to bone analysis, this may be acceptable in clinical situations. It must be emphasized that the situations experimentally simulated in this paper are extremely demanding (from $160^{\circ}$ down to $130^{\circ}$ for the angular view). They are nevertheless close to those encountered in rotational $-\mathrm{X}$ which, at the moment, is mainly devoted to vascular explorations. The search for efficient methods capable to fulfill the clinical requirements, while significantly reducing the irradiation, remains a true health problem and an open challenge. Our future work will focus on improving the present approach and extending its scope to fan-beam and cone-beam geometries. 


\section{Appendix}

Proof of Theorem 1. Using (8), (5) can be rewritten as

$$
H_{p}(\theta)=\sum_{x=0}^{N-1} \sum_{y=0}^{N-1} I(x, y) \sum_{q=0}^{p} c_{p q}\left(x_{\theta} y-y_{\theta} x\right)_{q} .
$$

Using the relationships [20]

$$
(x)_{r}=\sum_{k=0}^{r}(-1)^{r-k} S_{1}(r, k) x^{k} \quad \text { and } \quad x^{k}=\sum_{m=0}^{k}(-1)^{m} S_{2}(k, m)(-x)_{m} \text {, }
$$

we have

$$
\left(x_{\theta} y-y_{\theta} x\right)_{q}=\sum_{r=0}^{q} \sum_{s=0}^{r}(-1)^{q+s-r}\left(\begin{array}{l}
r \\
s
\end{array}\right) S_{1}(q, r) y_{\theta}{ }^{s} x_{\theta}^{r-s} \cdot \sum_{i=0}^{r-s}(-1)^{i} S_{2}(r-s, i)(-y)_{i} \sum_{j=0}^{s}(-1)^{j} S_{2}(s, j)(-x)_{j}
$$

Substitution of (A3) into (A1) yields

$$
\begin{aligned}
H_{p}(\theta) & =\sum_{q=0}^{p} \sum_{r=0}^{q} \sum_{s=0}^{r} \sum_{i=0}^{r-s} \sum_{j=0}^{s} \sum_{n=0}^{j} \sum_{m=0}^{i}(-1)^{q+s+i+j-r}\left(\begin{array}{l}
r \\
s
\end{array}\right) S_{1}(q, r) S_{2}(r-s, i) S_{2}(s, j) \\
& \cdot y_{\theta}^{s} x_{\theta}^{r-s} c_{p q} d_{i m} d_{j n} \sum_{x=0}^{N-1} \sum_{y=0}^{N-1} t_{n}(x) t_{m}(y) I(x, y) .
\end{aligned}
$$

Using (6) and making the change of variables $r^{\prime}=r-n-m, s^{\prime}=s-m$ in the last equation of the above expression, we can derive the result claimed in Theorem 1.

Proof of Proposition 2. To prove the proposition, we need to verify the following relation

$$
\sum_{q=r}^{l} c_{l q} d_{q r}=\delta_{l r} \quad \text { for } 0 \leq l, r \leq p
$$

Using (16) and (17), we have

$$
\sum_{q=r}^{l} c_{l q} d_{q r}=(-1)^{r} \frac{(2 r+1) \beta(r, N)(1-N)_{l}}{\beta(l, N)(1-N)_{r}} \sum_{q=r}^{l} \frac{(-1)^{q}(l+q) !}{(l-q) !(q+r+1) !(q-r) !} .
$$

For $l=r,(\mathrm{~A} 6)$ becomes

$$
c_{l l} d_{l l}=(-1)^{l}(2 l+1) \times \frac{(-1)^{l}(2 l) !}{(2 l+1) !}=1 .
$$

To prove (A6) for $r<l$, let 


$$
G(l, r, q)=(-1)^{q+1}\left(\begin{array}{c}
l+q+1 \\
l-q+1
\end{array}\right)\left(\begin{array}{c}
2 q \\
q-r
\end{array}\right) \frac{(l+1-q)(q-r)}{(l+q+1)(l+r+1)(l-r)}
$$

we have

$$
G(l, r, q+1)-G(l, r, k)=\frac{(-1)^{q}(l+q) !}{(l-q) !(q+r+1) !(q-r) !} .
$$

Thus

$$
\begin{aligned}
\sum_{q=r}^{l} \frac{(-1)^{q}(l+q) !}{(l-q) !(q+r+1) !(q-r) !} & =\sum_{q=r}^{l}[G(l, r, q+1)-G(l, r, q)] \\
& =G(l, r, l+1)-G(l, r, r)=0 .
\end{aligned}
$$

The proof is now complete. 


\section{References}

[1] H. Barrett, W. Swindle, Radiological Imaging: The Theory of Image Formation, Detection and Processing, Academic Press, New York, 1981.

[2] G.T. Herman, Image reconstruction from projections: The fundamentals of Computerized Tomography, Academic Press, New York, 1980.

[3] F. Natterer, The mathematics of Computerized Tomography, John Wiley \& Sons, New York, 1986.

[4] D. Salzman, A method of general moments for orienting 2D projections of unknown 3D objects, Comput. Vis. Graph. Image Process. 50 (1990) 129-135.

[5] A.B. Goncharev, Methods of integral geometry and recovering a function with compact support from its projections in unknown directions, Acta Appl. Math. 11 (1988) 213-222.

[6] P.M. Milanfar, W.C. Karl, A.S. Willsky, A moment-based variational approach to tomographic reconstruction, IEEE Trans. on Image Process. 5 (1996) 459-470.

[7] S. Basu, Y. Bresler, Uniqueness of tomography with unknown view angles, IEEE Trans. on Image Process. 9 (2000) 1094-1106.

[8] S. Basu, Y. Bresler, Feasibility of tomography with unknown view angles, IEEE Trans. on Image Process. 9 (2000) 1107-1122.

[9] T.J. Wang, T.W. Sze, The image moment method for the limited range CT image reconstruction and pattern recognition, Pattern Recognition, 34 (2001) 2145-2154.

[10] H.Z. Shu, J. Zhou, G.N. Han, L.M. Luo and J.L. Coatrieux, Image reconstruction from limited range projections using orthogonal moments, Pattern Recognition, 40 (2007) 670-680.

[11] R. Mukundan, S.H. Ong, P.A. Lee, Image analysis by Tchebichef moments, IEEE Trans. on Image Process. 10 (2001) 1357-1364.

[12] P.T. Yap, P. Raveendran, S.H. Ong, Image analysis by Krawtchouk moments, IEEE Trans. on Image Process. 12 (2003) 1367-1377.

[13] H.Q. Zhu, H.Z. Shu, J. Liang, L.M. Luo, J.L. Coatrieux, Image analysis by discrete orthogonal Racah moments, Signal Processing, 87 (2007) 687-708.

[14] H.Q. Zhu, H.Z. Shu, J. Liang, L.M. Luo, J.L. Coatrieux, Image analysis by discrete orthogonal dual-Hahn moments, Pattern Recognition Letters 28 (2007) 1688-1704.

[15] J. P. Guédon, N. Normand. The Mojette transform: The first ten years, in Discrete Geometry for Computer Imagery, Lecture Notes in Computer Science, Volume 3429/2005, Springer Berlin / Heidelberg, (2005) 79-91.

[16] G. Beylkin, Discrete Radon transform, IEEE Trans. on Acoustics Speech Signal Process. 35 (1987) 162-172.

[17] F. Matus, J. Flusser, Image representation via a finite radon transform, IEEE Trans. on Pattern Anal. Machine Intell. 15 (1993) 996-1106. 
[18] I. Svalbe, D. van der Spek, Reconstruction of tomographic images using analog projections and the digital radon transform, Linear Algebra and Its Applications, 39 (2001) 125-145.

[19] A. Kingston, I. Svalbe, Mapping between digital and continuous projections via the discrete radon transform in Fourier space, Digital Imaging Computing: Techniques and Applications, Sydney, Australia (2003) 263-272.

[20] V. Adamchik, On Stirling number and Euler sums, J. Comput. Appl. Math, 79 (1997) 119-130.

[21] L. A. Shepp, Y. Vardi, Maximum likelihood reconstruction for emission tomography, IEEE Trans. on Med. Imag., 1 (1982) 113-122.

[22] B. A. Ardekani, M. Braun, B. F. Hutton, et al, Minimum cross-entropy reconstruction of PET images using prior anatomical information, Phys. Med. Biol., 41 (1996) 2497-2517.

[23] A. H. Anderson, A. C. Kak, Simultaneous algebraic reconstruction technique (SART): a superior implementation of the ART algorithm, Ultrasonic Imaging, 6 (1984) 81-94.

[24] C.H. Teh, R.T. Chin, On image analysis by the method of moments, IEEE Trans. on Pattern Anal. Machine Intell. 10 (1988) 496-513.

[25] T.H. Farquhar, A. Chatziioannou, G. Chinn, M. Dahlbom, E.J. Hoffman, An investigation of filter choice for filtered back-projection reconstruction in PET, IEEE Trans. on Nucl. Sci., 45 (1998) $1133-1137$.

[26] T. Schaeffter, M. Grass, R. Proksa, et al, Real-Time Adaptive Filtering for Projection Reconstruction MR Fluoroscopy, IEEE Trans. on Med. Imag., 22 (2003) 75-81. 
TABLE 1: ERRORS BETWEEN THE IMAGE MOMENT VALUES OBTAINED WITH EQ. (6) AND IMAGE MOMENT VALUES ESTIMATED FROM DIGITAL PROJECTION MOMENTS WITH EQ. (11)

\begin{tabular}{c|c|c|c|c|}
\hline$n$ & 0 & 2 & 4 & 8 \\
\hline 0 & 0.0000 & -0.00008 & 0.00046 & -0.0001 \\
2 & -0.00027 & 0.0002 & -0.00003 & 0.00005 \\
4 & 0.00031 & -0.00012 & -0.0001 & -0.00006 \\
8 & 0.0000 & -0.000003 & -0.00004 & -0.00005 \\
\hline
\end{tabular}

TABLE 2. RECONSTRUCTION MSE (\%) OF FIG. 4 FOR DIFFERENT VALUES OF MAXIMUM ORDER OF MOMENTS $M$

\begin{tabular}{|c|c|c|c|c|}
\hline $\mathrm{Method}^{M}$ & 5 & 10 & 15 & 20 \\
\hline Legendre & 11.2860 & 8.4279 & 5.9729 & 5.4071 \\
\hline $\begin{array}{l}\text { Tchebichef } \\
\text { (IDRT) }\end{array}$ & 9.0753 & 6.5466 & 3.6704 & 3.0925 \\
\hline $\begin{array}{c}\text { Tchebichef } \\
\text { (FBP) }\end{array}$ & 10.5623 & 7.0158 & 4.9478 & 3.9878 \\
\hline FBP & \multicolumn{4}{|c|}{17.8945} \\
\hline SART & \multicolumn{4}{|c|}{18.9854} \\
\hline MLEM & \multicolumn{4}{|c|}{5.5214} \\
\hline MXE & \multicolumn{4}{|c|}{5.4801} \\
\hline
\end{tabular}

TABLE 3 THE MEAN GREY LEVELS OF THREE RECONSTRUCTED ELLIPSES E1, E2 AND E3.

\begin{tabular}{|c|c|c|c|c|}
\hline$M$ & 5 & 10 & 15 & 20 \\
\hline Method & \multicolumn{5}{|c|}{$0.739: 2.024: 2.744$} \\
\hline FBP & \multicolumn{4}{|c|}{$0.669: 2.005: 2.736$} \\
\hline SART & \multicolumn{4}{|c|}{$1.003: 2.725: 3.689$} \\
\hline MLEM & \multicolumn{5}{|c|}{$1.003: 2.742: 3.699$} \\
\hline Legendre & $0.978: 2.241: 3.035$ & $1.008: 2.251: 3.205$ & $1.006: 2.455: 3.437$ & $0.995: 2.529: 3.545$ \\
\hline Tchebichef(IDRT) & $0.994: 2.421: 3.257$ & $1.004: 2.498: 3.347$ & $1.003: 2.774: 3.701$ & $1.002: 2.905: 3.875$ \\
\hline Tchebichef(FBP) & $0.982: 2.333: 3.185$ & $1.006: 2.412: 3.265$ & $1.004: 2.678: 3.521$ & $0.997: 2.716: 3.803$ \\
\hline
\end{tabular}

TABLE 4. MAXIMUM ORDERS OF MOMENTS REQUIRED FOR THE METHODS BASED ON LEGENDRE POLYNOMIALS AND TCHEBICHEF POLYNOMIALS TO GET SIMILAR VALUES OF MSE

\begin{tabular}{|c|c|c|c|c|}
\cline { 2 - 5 } \multicolumn{1}{c|}{} & \multicolumn{4}{c|}{ MSE(\%) values and corresponding maximum order of moments $M$} \\
\hline Legendre & $9.3100(M=8)$ & $8.4279(M=10)$ & $5.9729(M=15)$ & $5.4071(M=20)$ \\
\hline $\begin{array}{c}\text { Tchebichef } \\
\text { (IDRT) }\end{array}$ & $9.0753(M=5)$ & $8.4466(M=8)$ & $5.8704(M=11)$ & $5.2104(M=12)$ \\
\hline $\begin{array}{c}\text { Tchebichef } \\
\text { (FBP) }\end{array}$ & $9.1024(M=5)$ & $8.5277(M=8)$ & $6.0023(M=11)$ & $5.4128(M=12)$ \\
\hline
\end{tabular}


TABLE 5. RECONSTRUCTION MSE (\%) USING LEGENDRE MOMENTS AND TCHEBICHEF MOMENTS FOR DIFFERENT VALUES OF CUT-OFF FREQUENCY WITH $M=15$.

\begin{tabular}{|c|c|c|c|c|}
\hline Method & 0.5 & 0.7 & 0.9 & 1 \\
\hline FBP & 19.427 & 18.729 & 18.457 & 18.556 \\
\hline Legendre & 7.866 & 7.385 & 7.258 & 7.265 \\
\hline Tchebichef (IDRT) & 5.123 & 5.123 & 5.123 & 5.123 \\
\hline Tchebichef (FBP) & 6.874 & 6.474 & 6.001 & 6.311 \\
\hline
\end{tabular}

TABLE 6. RECONSTRUCTION MSE (\%) OF FIG. 5 FOR DIFFERENT VALUES OF MAXIMUM ORDER OF MOMENTS $M$ WITH THE CUT-OFF FREQUENCY $\xi_{\text {MAX }}=0.7$.

\begin{tabular}{|c|c|c|c|c|}
\hline Method $^{M}$ & 5 & 10 & 15 & 17 \\
\hline Legendre & 12.543 & 9.318 & 7.385 & 42.546 \\
\hline Tchebichef(IDRT) & 9.897 & 7.691 & 5.123 & 30.444 \\
\hline Tchebichef(FBP ) & 11.462 & 8.223 & 6.474 & 38.187 \\
\hline FBP & \multicolumn{4}{|c|}{18.729} \\
\hline SART & \multicolumn{4}{|c|}{19.965} \\
\hline MLEM & \multicolumn{4}{|c|}{9.542} \\
\hline MXE & \multicolumn{4}{|c|}{9.398} \\
\hline
\end{tabular}

TABLE 7. RECONSTRUCTION MSE (\%) OF FIG. 6 USING FBP METHOD, THE METHOD BASED ON LEGENDRE MOMENTS AND OUR METHOD WITH MAXIMUM ORDER $M=15$ FOR DIFFERENT VALUES OF

$\alpha$.

\begin{tabular}{|c|c|c|c|c|c|}
\hline$\alpha$ & 0 & 10 & 15 & 20 & 25 \\
\hline Method & 25.052 & 32.731 & 36.902 & 41.614 & 45.072 \\
\hline FBP & 25.052 & 27.733 & 28.813 & 31.681 & 35.402 \\
\hline Legendre & 19.524 & 21.011 & 22.412 & 25.314 & 29.768 \\
\hline
\end{tabular}

TABLE 8 THE DIFFERENCE OF MEAN GREY LEVELS OF REGIONS OF INTEREST BETWEEN RECONSTRUCTED RESULTS SHOWN IN FIG.6 AND ORIGINAL IMAGE IN FIG.6 (A).

\begin{tabular}{|c|c|c|c|c|c|}
\hline$\alpha$ & 0 & 10 & 15 & 20 & 25 \\
\hline Method & 2.3291 & 18.3010 & 26.7710 & 34.6410 & 41.9750 \\
\hline FBP & 2.3291 & 3.7642 & 5.8551 & 8.9654 & 10.1023 \\
\hline Tchebichdre & 1.9283 & 2.4501. & 4.6709 & 6.4441 & 9.0123 \\
\hline
\end{tabular}




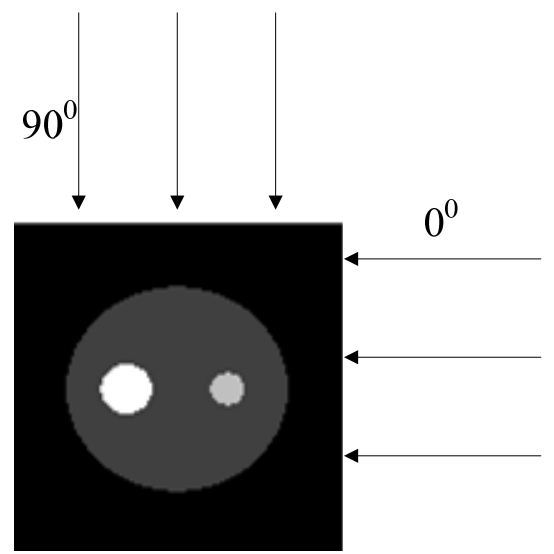

Figure 1 simulated phantom and the sample directions at $0^{\circ}$ and $90^{\circ}$. 


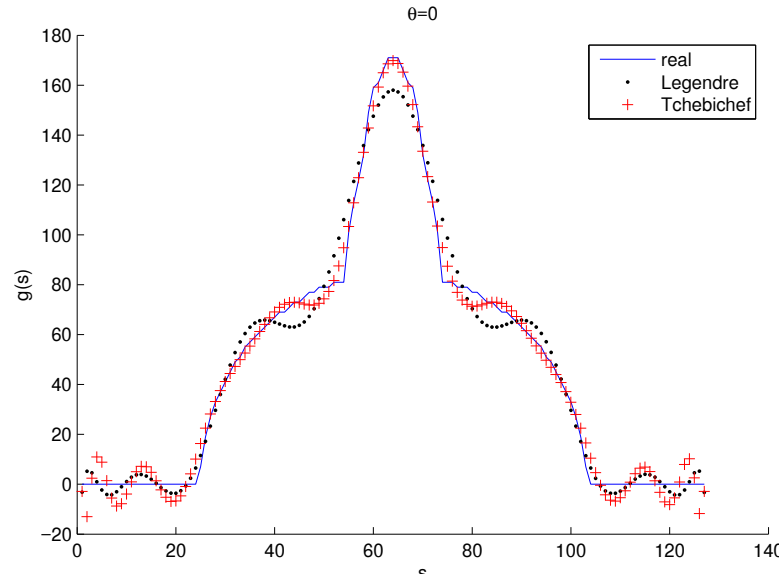

(a)
$\theta=60$

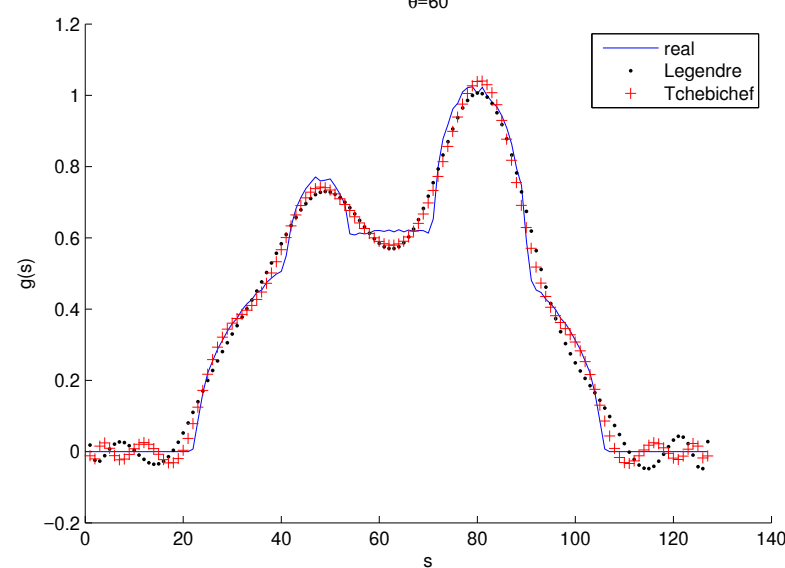

$\underset{\theta=130}{(c)}$

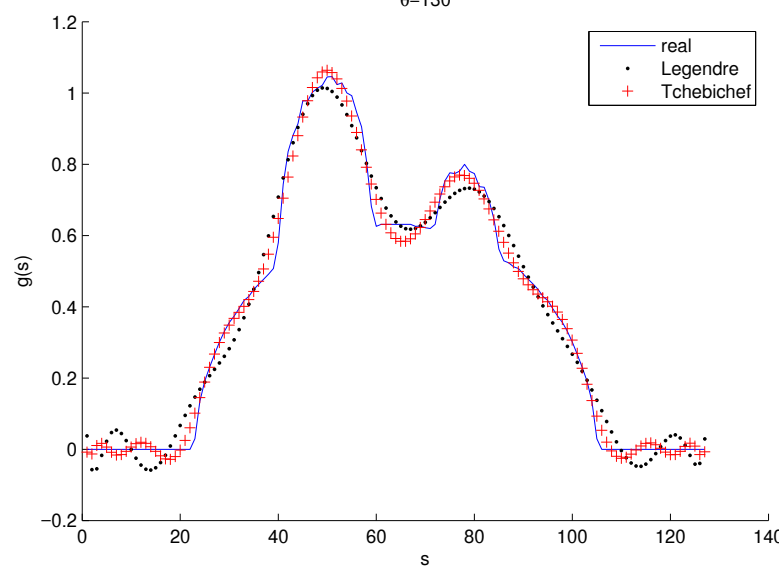

(e)

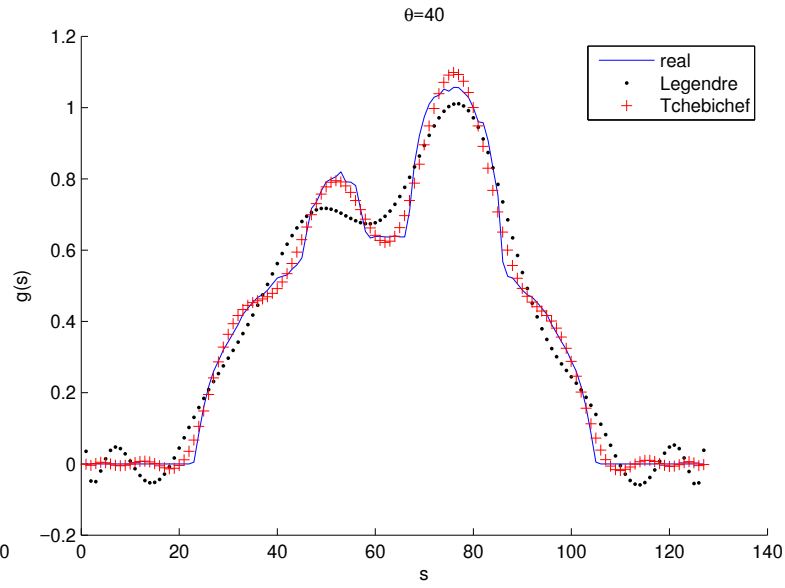

(b)

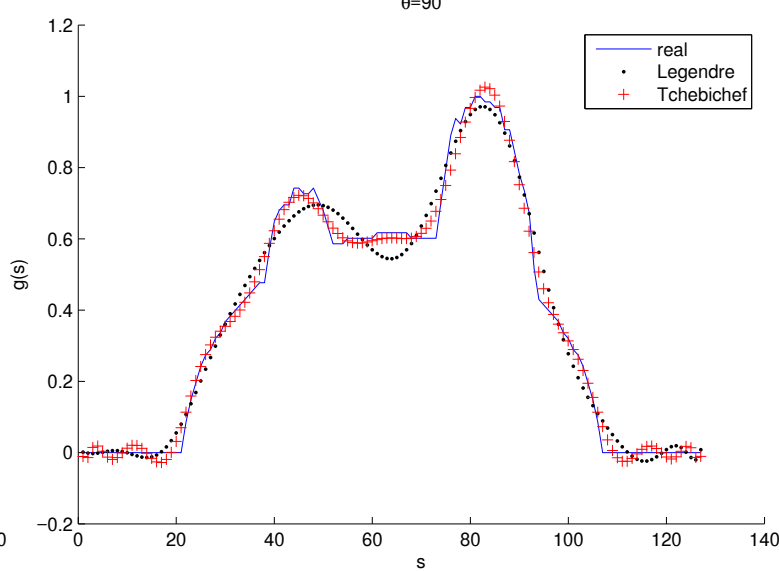

(d)

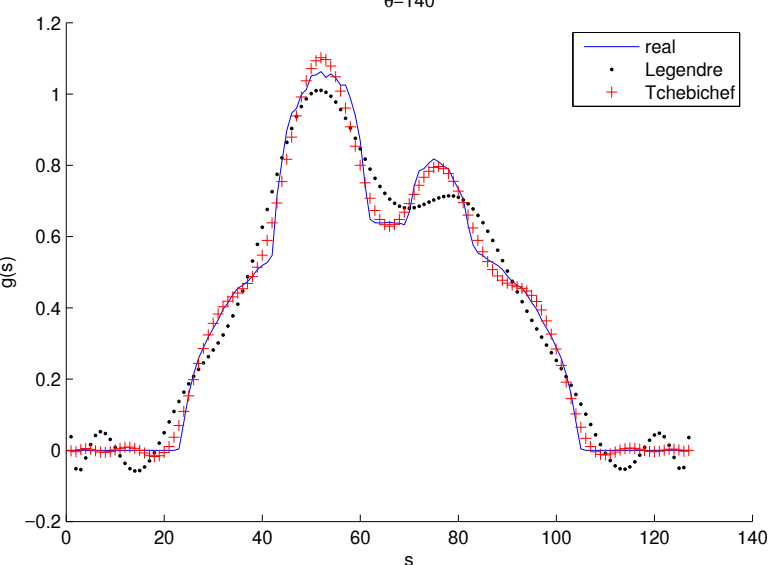

(f)

Figure 2 Results when all the projections are known. The projections are compared at different views: $0^{\circ}, 40^{\circ}, 60^{\circ}, 90^{\circ}, 130^{\circ}$ and $140^{\circ}$. Image moments of order up to 20 are used. (solid line: original projections; cross: projections estimated from Tchebichef moments; point: projections estimated from Legendre moments) 


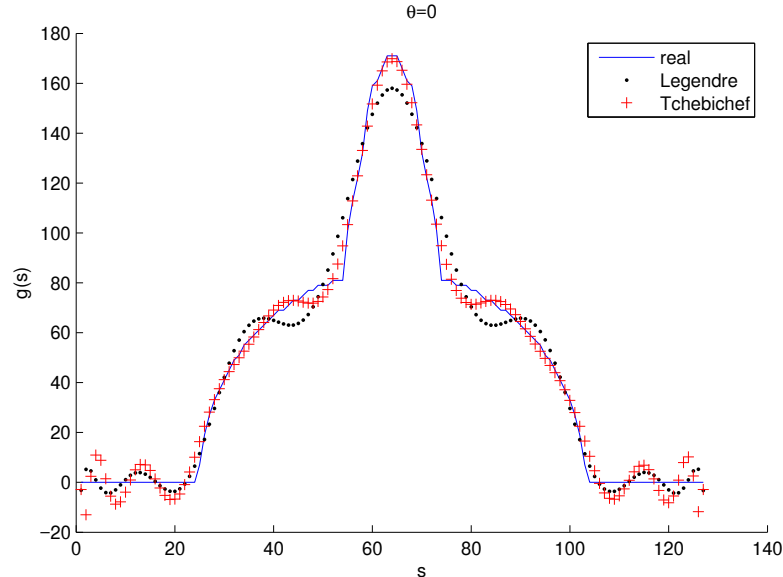

(a)

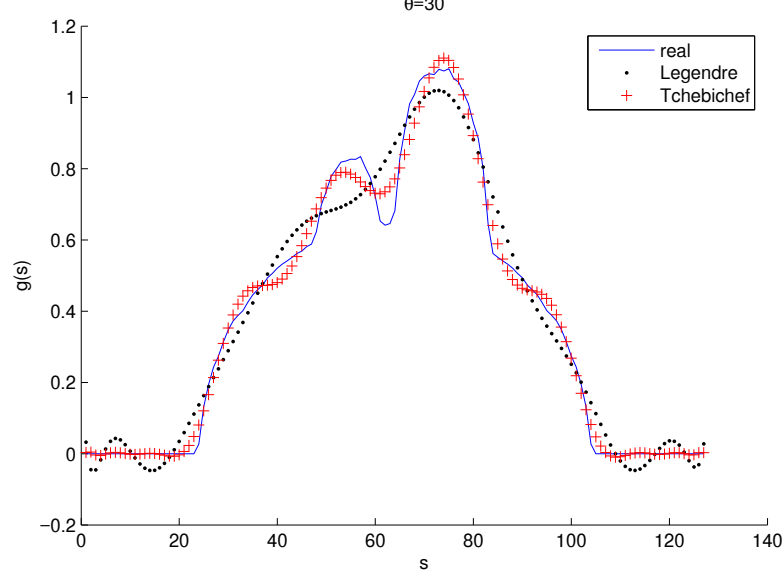

$\underset{\theta=170}{(\mathrm{c})}$

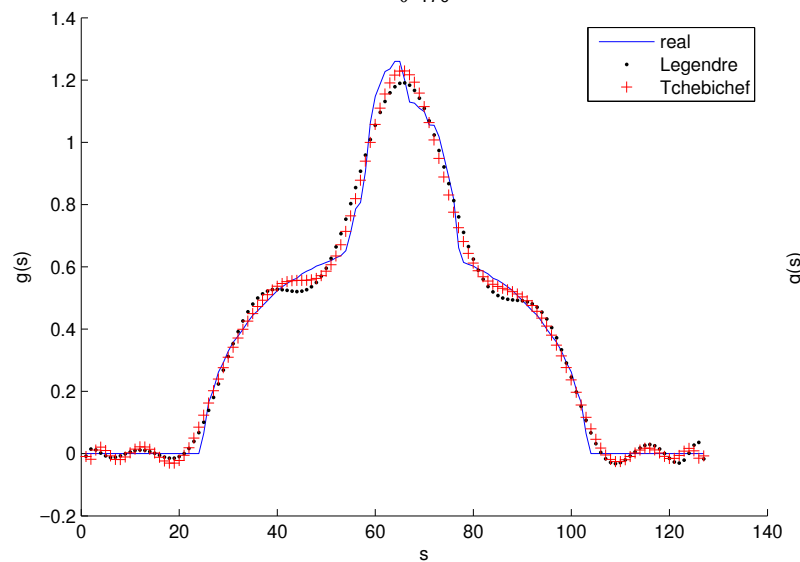

(e)

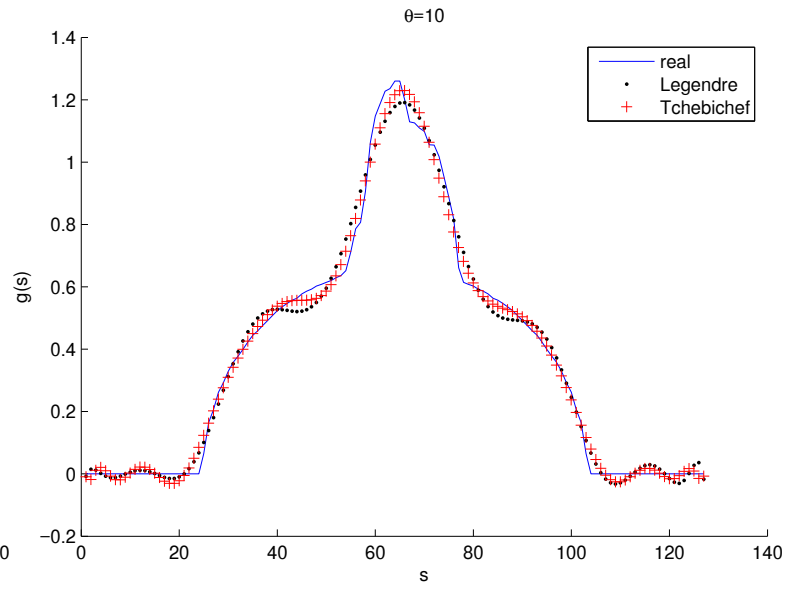

(b)

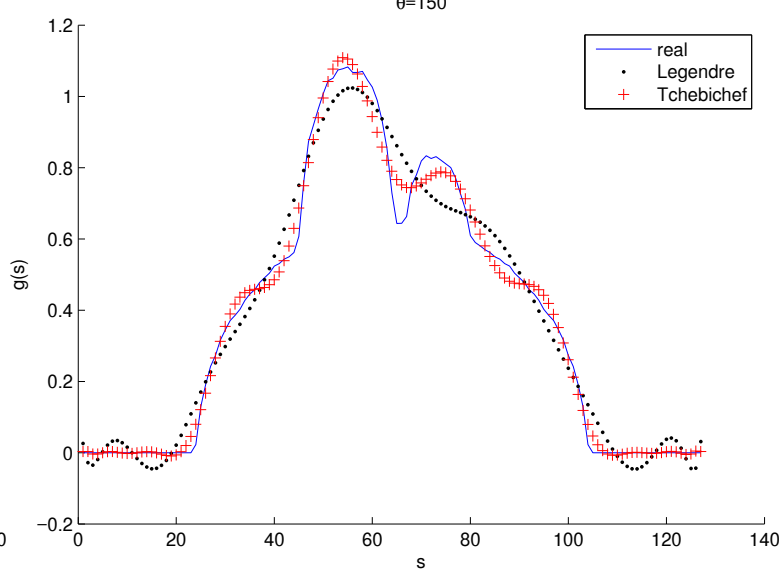

(d)

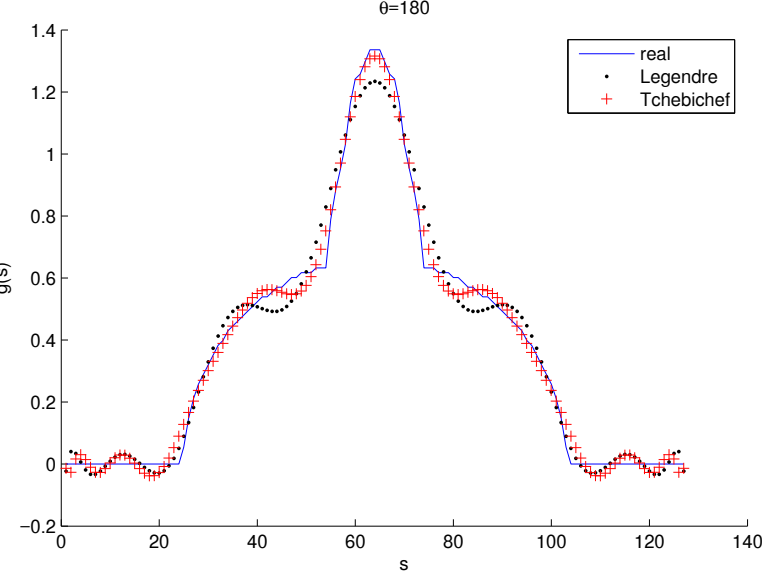

(f)

Figure 3 Results when only the projections in the range of $25^{0} \leq \theta \leq 155^{0}$ are known. The projections are compared at unknown views: $0^{\circ}, 10^{\circ}, 30^{\circ}, 150^{\circ}, 170^{\circ}$ and $180^{\circ}$. Image moments of order up to 20 are used. (solid line: original projections; cross: projections estimated from Tchebichef moments; point: projections estimated from Legendre moments) 


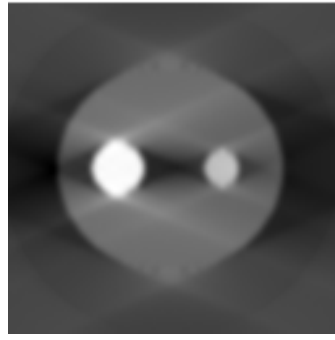

(a)

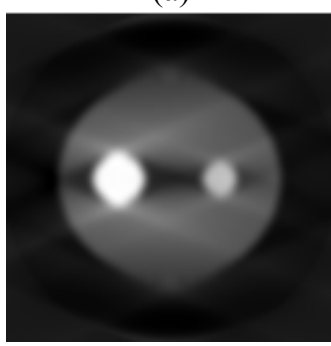

(e)

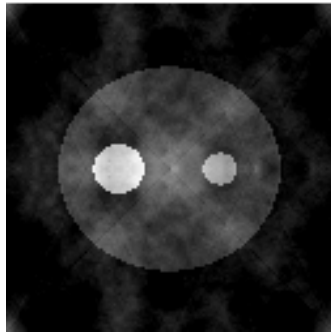

(f) $\quad M=5$

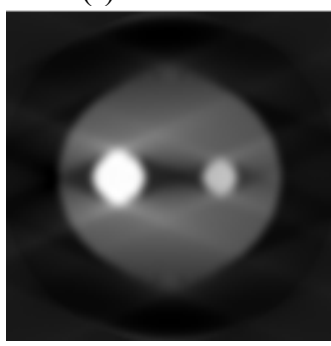

(g)

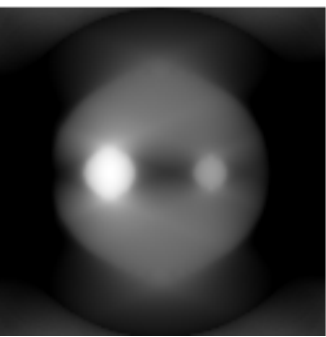

(b)

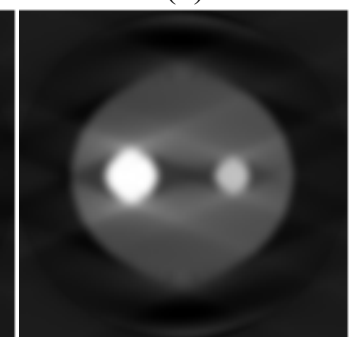

$M=10$

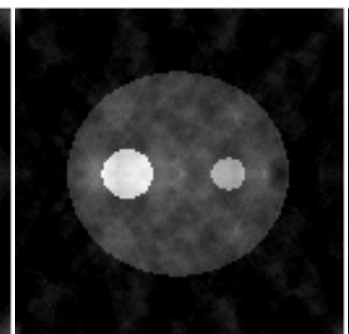

$M=10$

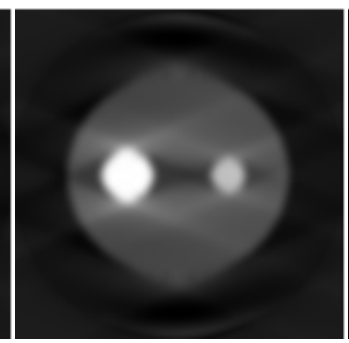

$M=10$

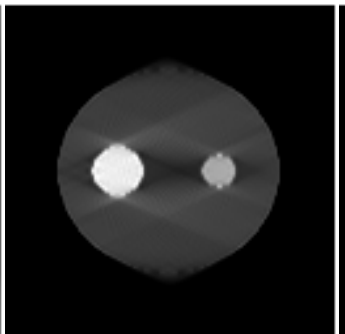

(c)

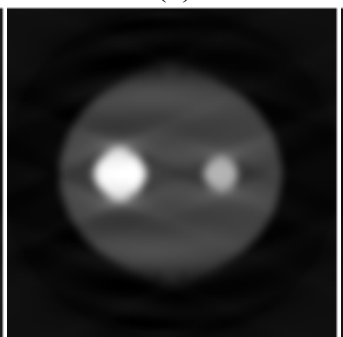

$M=15$

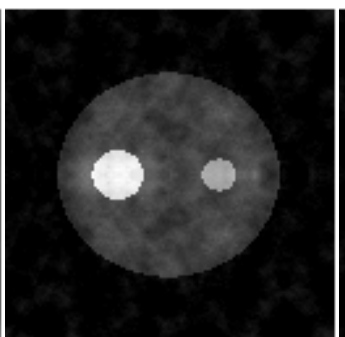

$M=15$

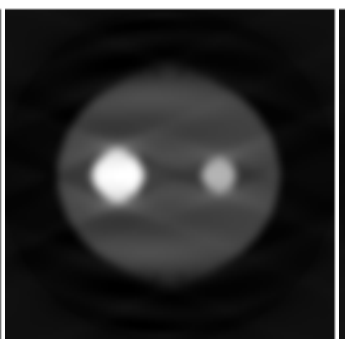

$M=15$

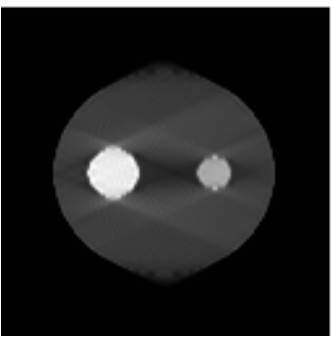

(d)

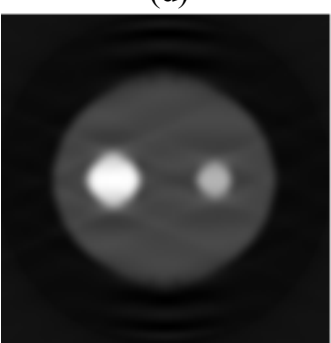

$M=20$

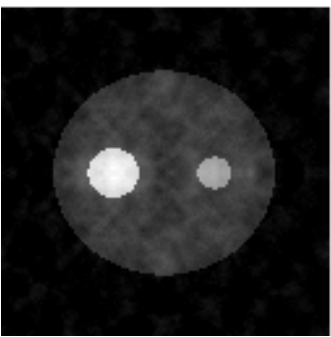

$M=20$

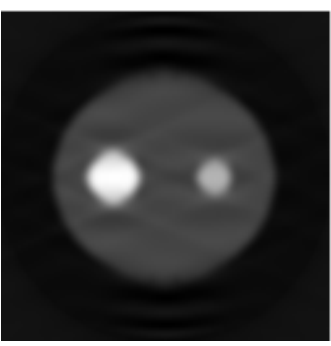

$M=20$

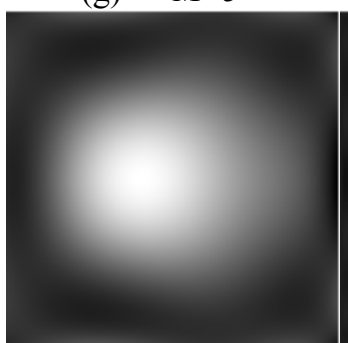

(h) $\quad M=5$

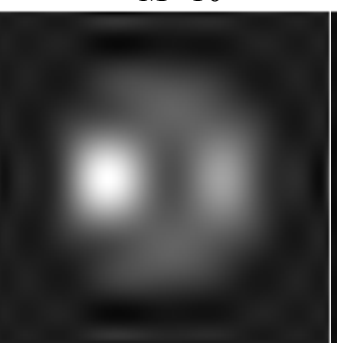

$M=10$

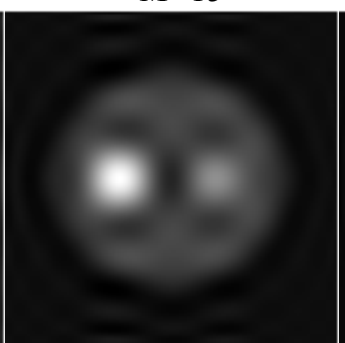

$M=15$

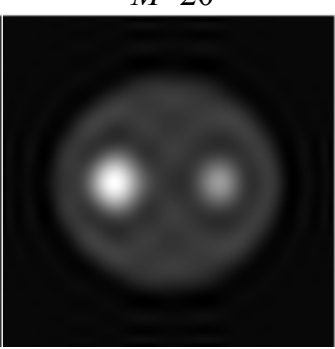

$M=20$

Figure 4 Reconstruction results from incomplete projections $\left(25^{0}-155^{\circ}\right)$. (a)-(d) Reconstruction using FBP, SART, MLEM, MXE method respectively; (e) Reconstructions using Legendre moments with different values of maximum order $M$; (f) Reconstructions using Tchebichef moments with different values of $M$, where the inverse discrete Radon transform (IDRT) is used in the reconstruction process; (g) Reconstruction using Tchebichef moments with different values of $M$, where the FBP is used in reconstruction process; (h) Reconstruction using the inverse moment transform defined by (6) with different values of $M$. 


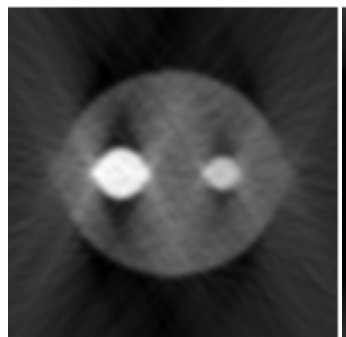

(a)

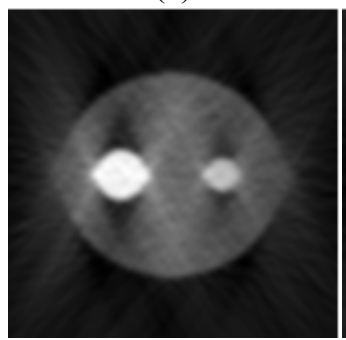

(e) $\quad M=5$

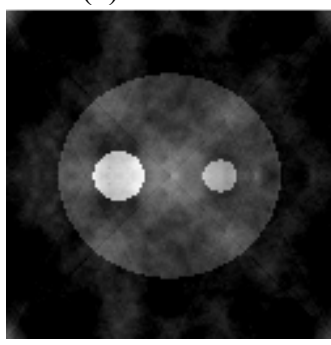

(f) $\quad M=5$

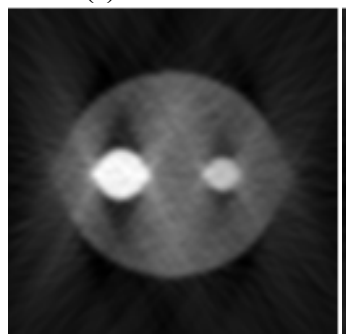

(g) $\quad M=5$

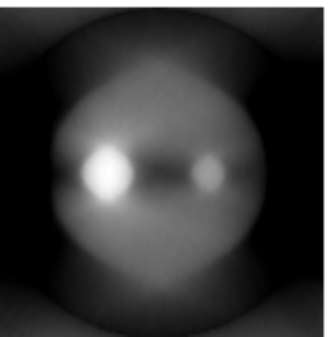

(b)

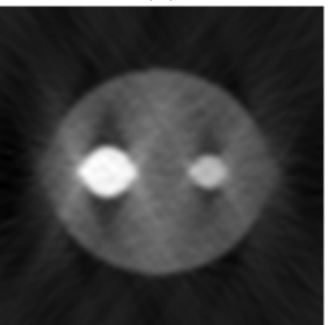

$M=10$

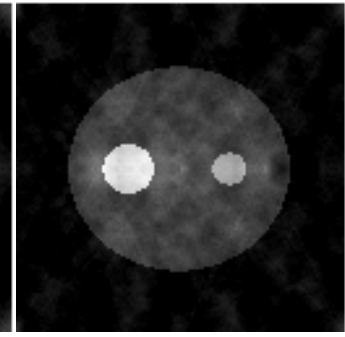

$M=10$

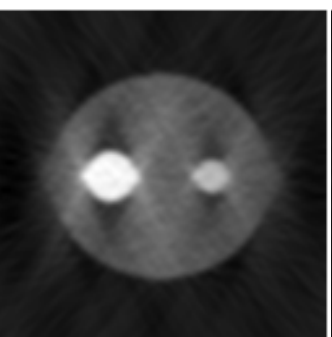

$M=10$

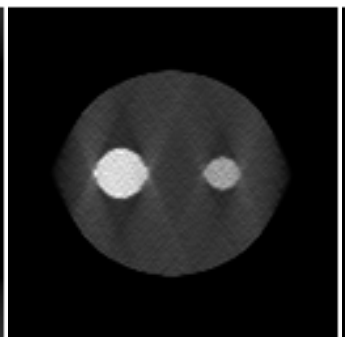

(c)

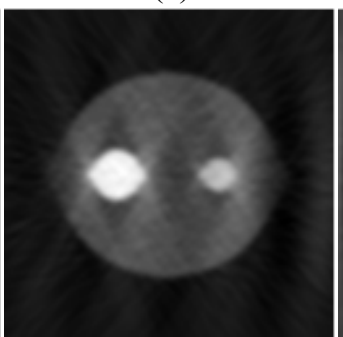

$M=15$

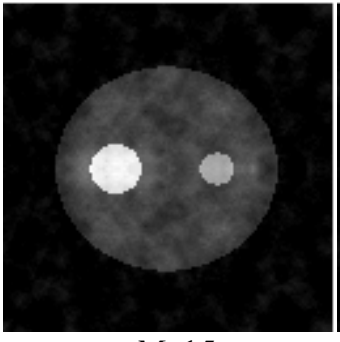

$M=15$

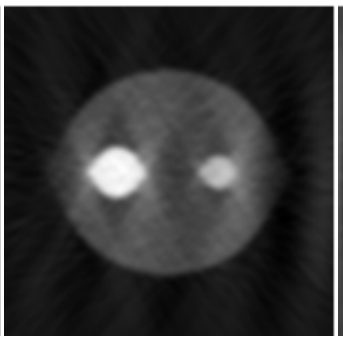

$M=15$

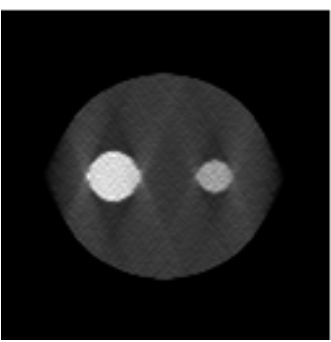

(d)

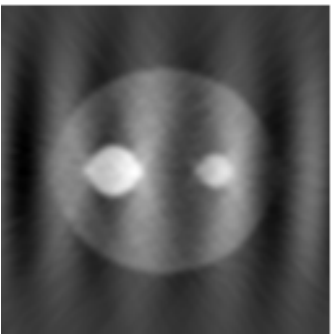

$M=17$

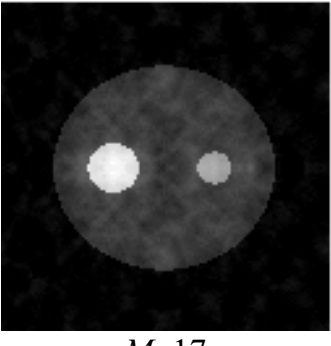

$M=17$

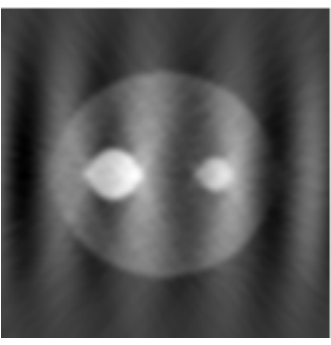

$M=17$

Figure 5 Reconstruction from incomplete projections $\left(25^{\circ}-155^{\circ}\right)$ with Poisson noise when cut-off frequency $\xi_{\max }=0.7$. (a)-(d) Reconstruction using FBP, SART, MLEM, MXE method respectively; (e) Reconstructions using Legendre moments with different values of $M$; (f) Reconstructions using Tchebichef moments with different values of $M$ where the inverse discrete Radon transform (IDRT) is used in the reconstruction process; (g) Reconstruction results using Tchebichef moments with different values of $M$ where the FBP is used in reconstruction process. 


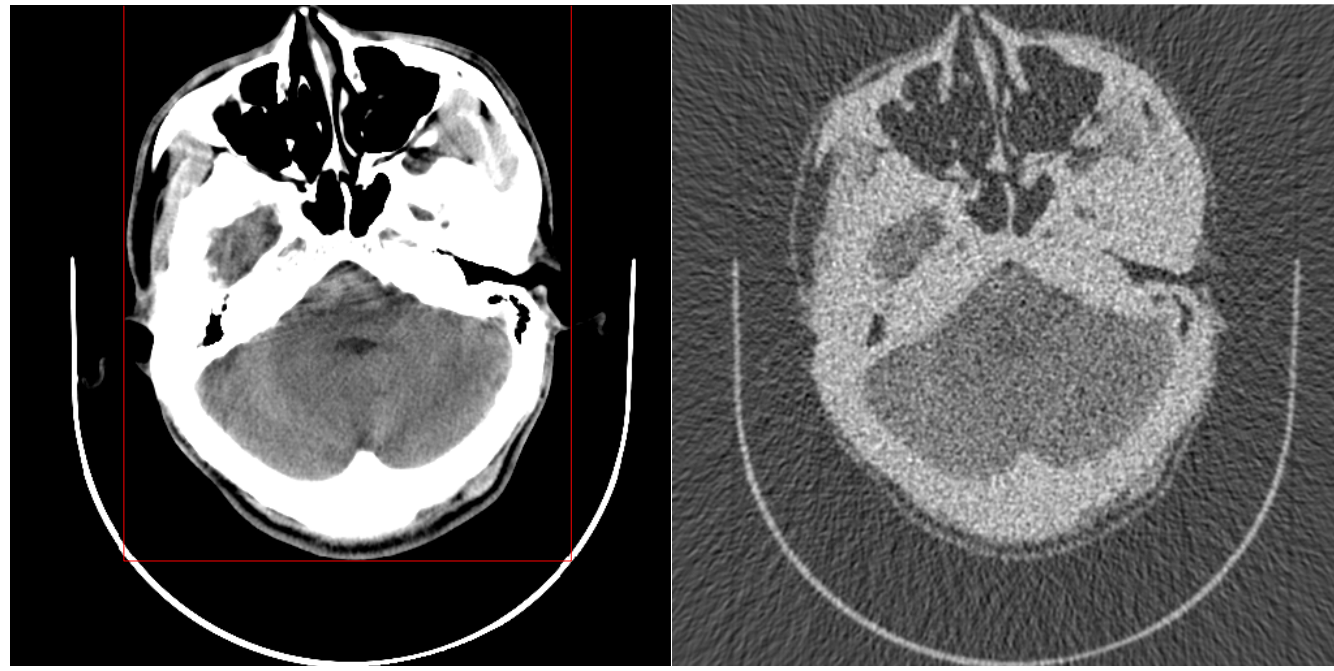

(a)

(b) $\alpha=0$

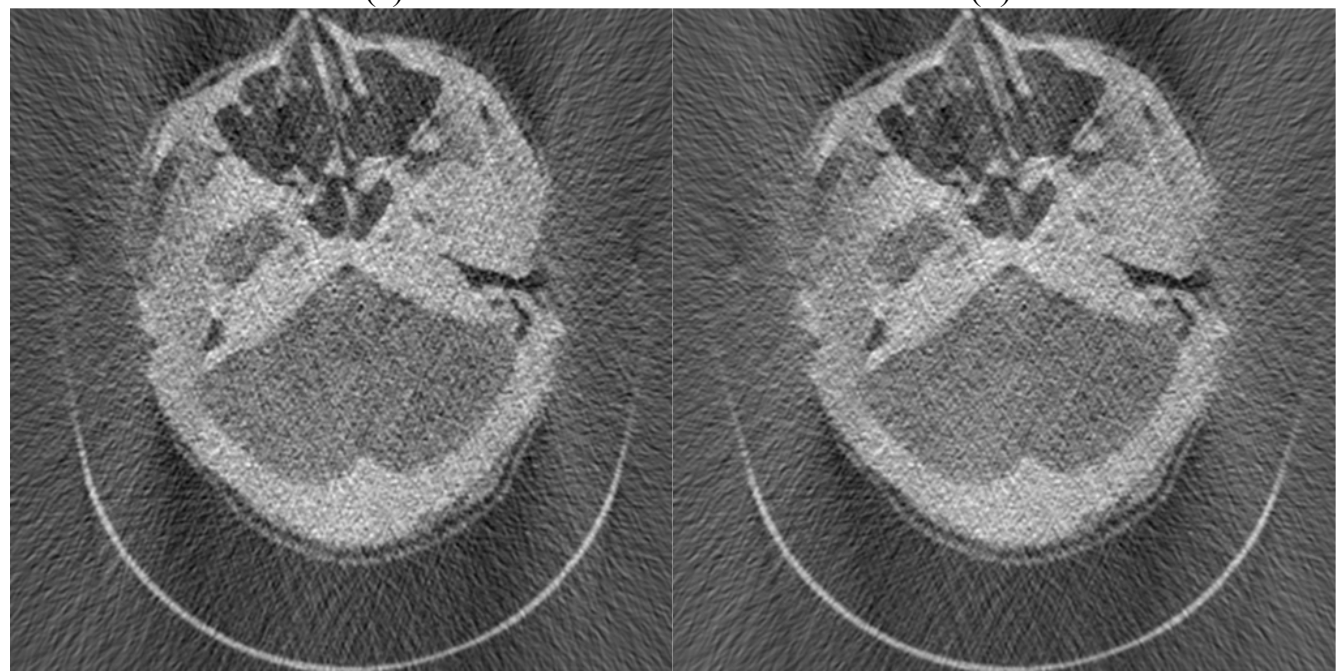

(c) $\alpha=10$

(d) $\alpha=15$

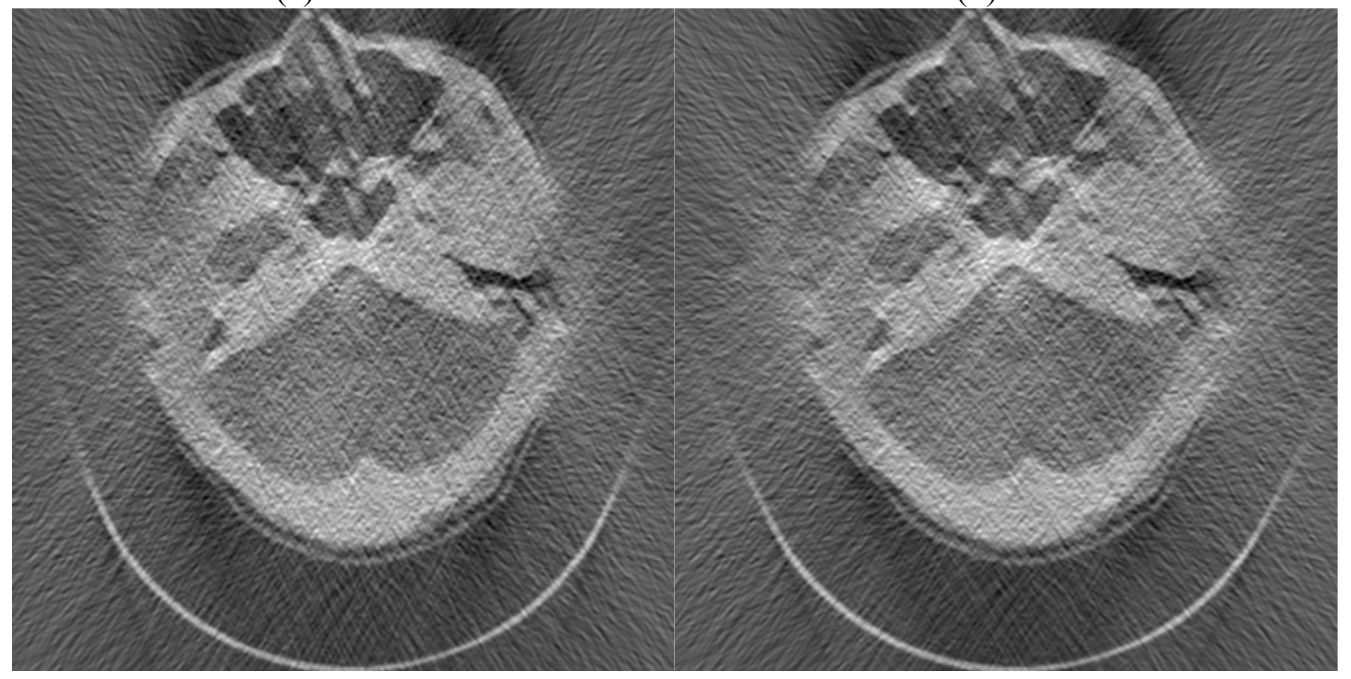

(e) $\alpha=20$

(f) $\alpha=25$ 


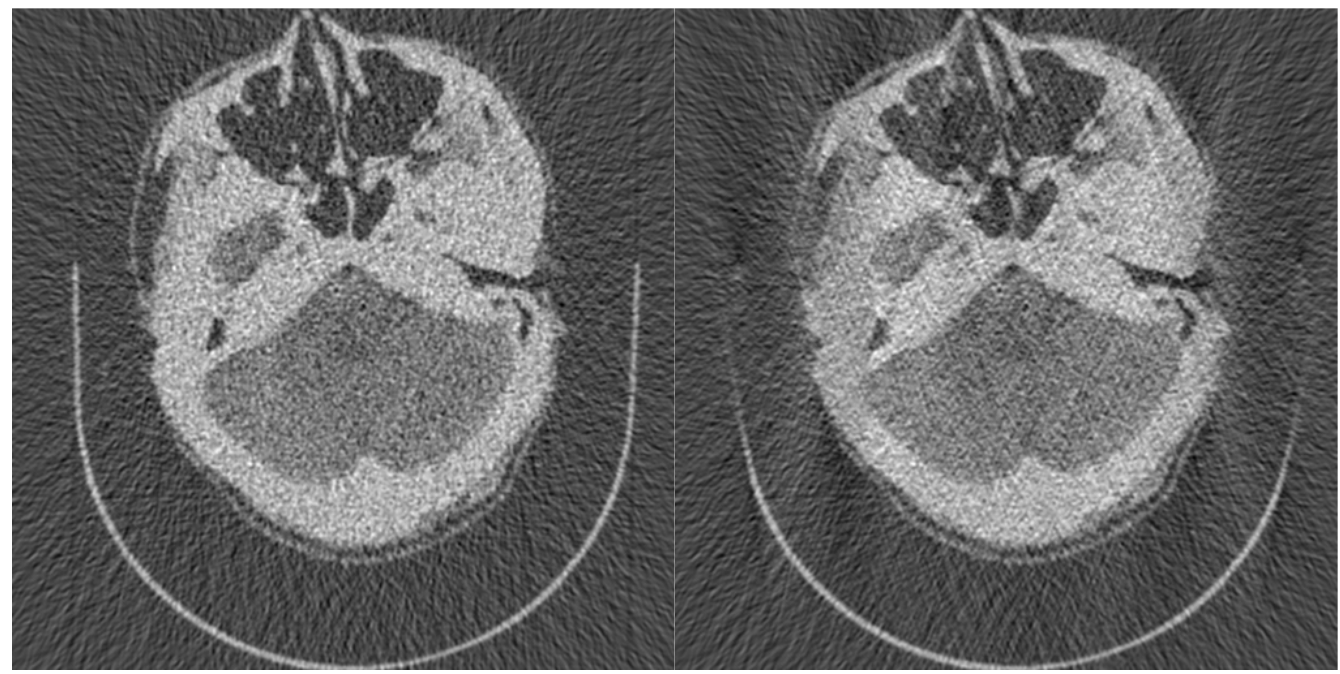

(g) $\alpha=0$

(h) $\alpha=10$

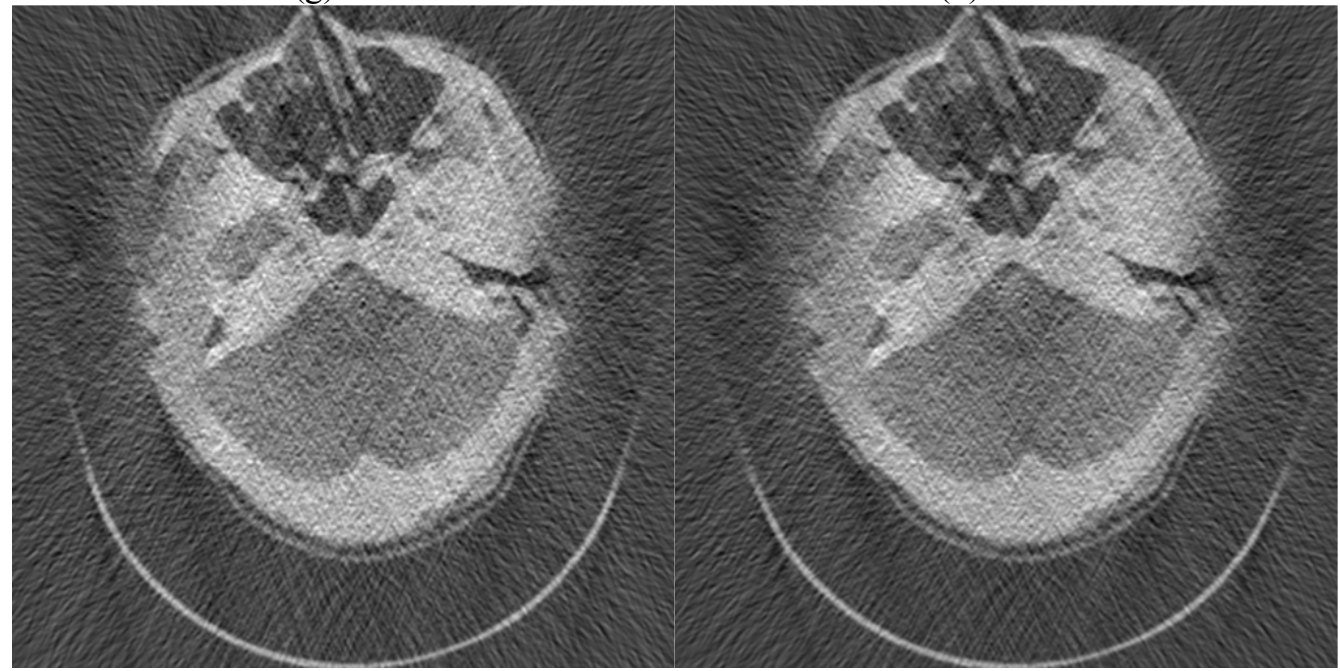

(i) $\alpha=15$

(j) $\alpha=20$

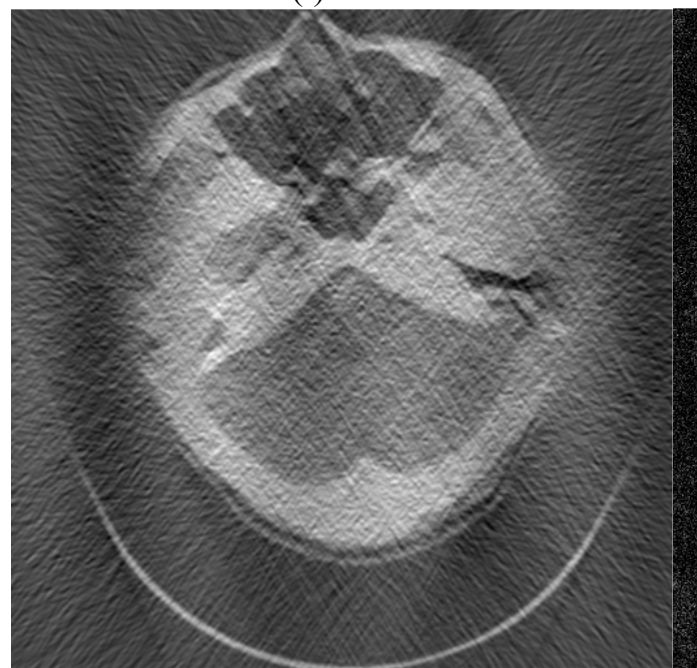

(k) $\alpha=25$

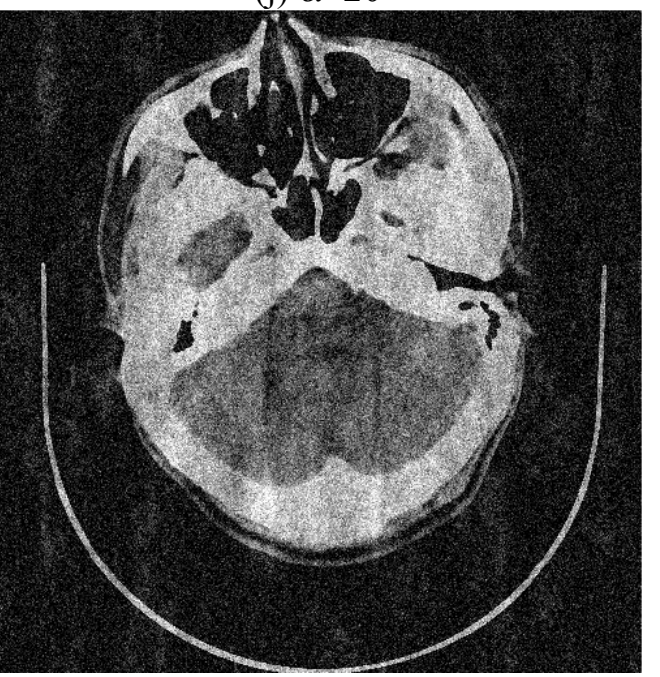

(1) $\alpha=0$ 


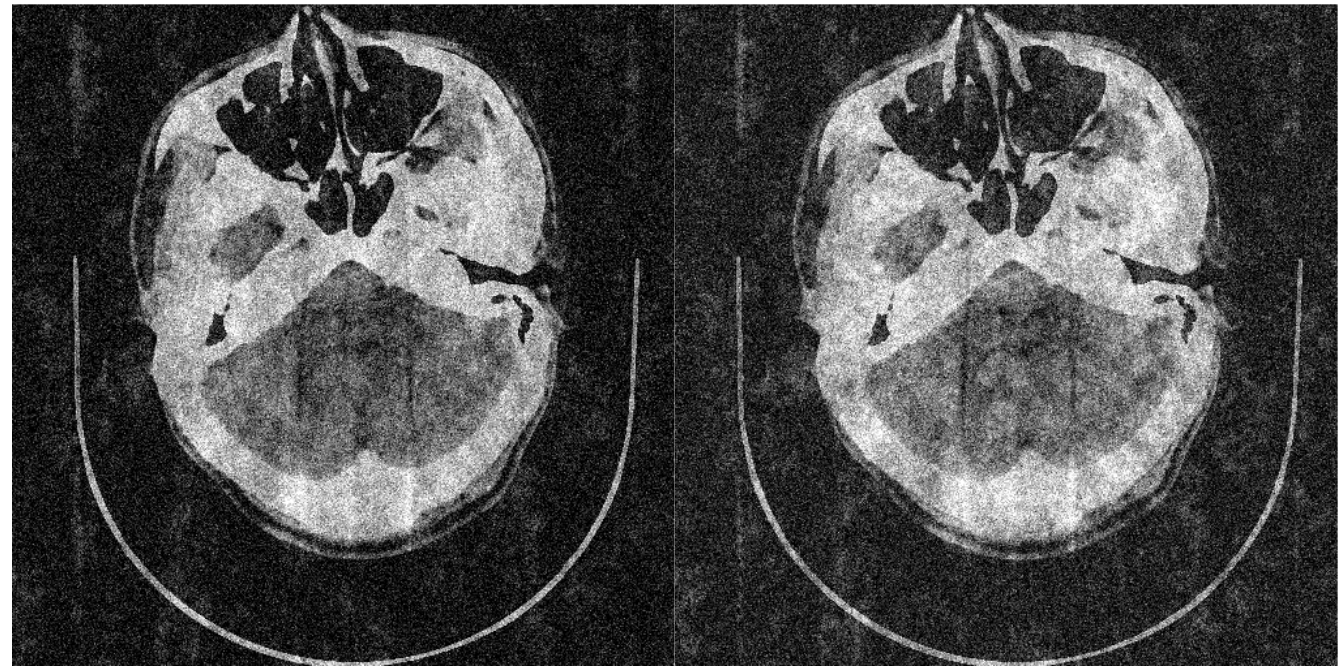

(m) $\alpha=10$

(n) $\alpha=15$

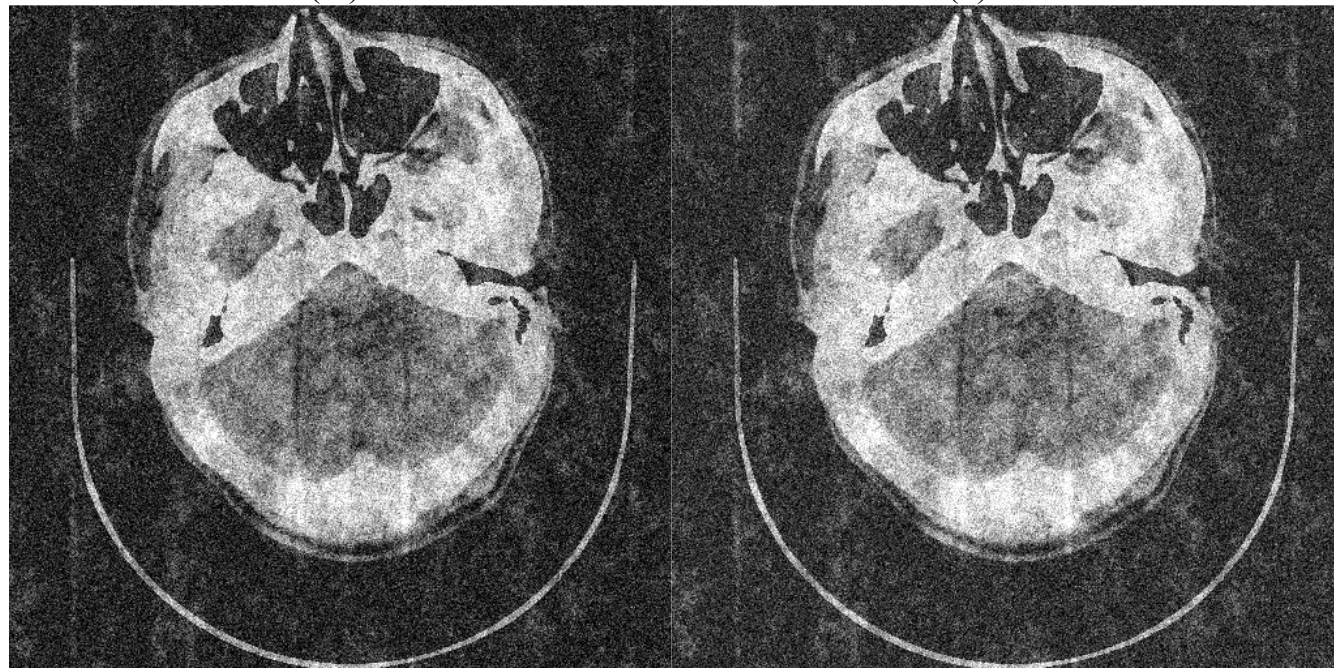

(o) $\alpha=20$

(p) $\alpha=25$

Figure 6 Reconstruction results of "pseudo-real" data with Poisson noise for $\alpha=0,10,15,20,25$ respectively. The size of image is $509 \times 509$ and maximum order $M$ is 15 . (a) Original image; (b)-(f) Reconstruction results using FBP method; $(\mathrm{g})-(\mathrm{k})$ Reconstruction results using the method based on Legendre moments; (1)-(p) Reconstruction results using the proposed method where the IDRT is used in the reconstruction process. 